\title{
WIYN Open Cluster Study. XXXVI. Spectroscopic Binary Orbits in NGC 188
}

\author{
Aaron M. Geller ${ }^{1}$ and Robert D. Mathieu ${ }^{1}$, \\ Department of Astronomy, University of Wisconsin - Madison, WI 53706 USA \\ and \\ Hugh C. Harris \\ United States Naval Observatory, Flagstaff, AZ, 86001, USA \\ and \\ Robert D. McClure \\ Dominion Astrophysical Observatory - Herzberg Institute of Astrophysics - National Research Council \\ 5071 W. Saanich Road, Victoria, BC V9E 2E7 Canada
}

\begin{abstract}
We present 98 spectroscopic binary orbits resulting from our ongoing radial-velocity survey of the old ( 7 Gyr) open cluster NGC 188. All but 13 are high-probability cluster members based on both radial-velocity and proper-motion membership analyses. 15 of these member binaries are double lined. Our stellar sample spans a magnitude range of $10.8 \leq \mathrm{V} \leq 16.5\left(1.14-0.92 \mathrm{M}_{\odot}\right)$ and extends spatially to $17 \mathrm{pc}(\sim 13$ core radii). All of our binary orbits have periods ranging from a few days to on the order of $10^{3}$ days, and thus are hard binaries that dynamically power the cluster. For each binary, we present the orbital solutions and place constraints on the component masses. Additionally, we discuss a few binaries of note from our sample, identifying a likely blue straggler - blue straggler binary system (7782), a double-lined binary with a secondary star which is under-luminous for its mass (5080), two potential eclipsing binaries (4705 and 5762), and two binaries which are likely members of a quadruple system (5015a and 5015b).

Subject headings: (galaxy:) open clusters and associations: individual (NGC 188) - (stars:) binaries: spectroscopic - (stars:) blue stragglers
\end{abstract}

\section{Introduction}

Within an open cluster, dynamical interactions with hard binarie:2 provide energy to the cluster, and can foster a complex interplay of stellar evolution, stellar dynamical exchanges, mass transfer, and even

\footnotetext{
${ }^{1}$ Visiting Astronomer, Kitt Peak National Observatory, National Optical Astronomy Observatory, which is operated by the Association of Universities for Research in Astronomy (AURA) under cooperative agreement with the National Science Foundation.

${ }^{2} \mathrm{~A}$ hard binary is defined as having an internal energy that is much greater than the energy of the relative motion of a single star moving within the cluster (Heggie 1974). For solar mass stars in a cluster with a one-dimensional velocity dispersion equal to $1 \mathrm{~km} \mathrm{~s}^{-1}$, all hard binaries have periods less than $\sim 10^{5}$ days.
} 
stellar collisions. Such interactions have the potential to result in the formation of "anomalous" stars that defy standard stellar evolutionary theory, such as blue stragglers (BSs). Recent $N$-body simulations (e.g., Hurley et al. 2005) are beginning to illuminate the likely formation mechanisms of such anomalous stars within open clusters, and it has become clear that the binary population plays a significant role. Detailed studies of open cluster binary populations are critical to constrain such models so that we can study cluster evolution as well as the formation mechanisms of anomalous stars. Furthermore, accurate and comprehensive surveys of binary populations are essential for our understanding of the onset of mass transfer, tidal interactions, initial and present-day mass functions, stellar dynamics, and even star formation processes.

Radial-velocity (RV) surveys offer an efficient way to identify singl 3 and binary open cluster members as well as to solve for binary orbital solutions. Open clusters are ideally suited for such surveys as they offer a coeval sample of stars that are generally easily accessible through ground-based observations using even modest-sized telescopes. Spectroscopic binary surveys have been carried out for a few well known clusters (e.g., Hyades Debernardi et al. (2000); Praesepe, Mermilliod \& Mavor (1999), Abt \& Willmarth (1999), Debernardi et al. (2000); Pleiades, Mermilliod et al. (1992); and M67; Mathieu, Latham \& Griffin (1990)). Today, the advent of multi-object spectrographs permits surveys of larger stellar samples in more distant open clusters, allowing us to explore binary populations as a function of age, stellar density, metallicity and stellar mass.

We present 98 binary orbits in the old ( 7 Gyr) open cluster NGC 188, derived from our ongoing RV survey of the cluster, covering a magnitude range of $10.8 \leq \mathrm{V} \leq 16.5\left(1.14-0.92 \mathrm{M}_{\odot}\right)$, a $1^{\circ}$ diameter region on the sky (roughly 13 core radi 4 ) and, for some binaries, a timespan of up to thirty years. This survey of NGC 188 is part of the WIYN Open Cluster Study (WOCS; Mathieu 2000). Our detectable binaries all have periods ranging from a few days to on the order of $10^{3}$ days. Given an internal velocity dispersion of $0.64 \pm 0.04 \mathrm{~km}$ $\mathrm{s}^{-1}$ (Geller et al. 2008, hereafter Paper 1), these binaries constitute much of the hard-binary population that dynamically powers the cluster. In Paper 1, we describe our observations, data reduction and the precision of our measurements. We also provide RV membership probabilities $\left(\mathrm{P}_{R V}\right)$ for stars observed $\geq 3$ times and identify RV variable stars. In this second paper in the series, we present our complete current RV database on the cluster (Section 2). In Section 3, we provide the 70 single-lined (SB1) and 15 double-lined (SB2) binary cluster-member orbital solutions derived from this survey. For each binary, we provide the plotted orbital solution, tabulated orbital parameters, and constraints on the component masses. In Section 4 we discuss a few binaries of note, including a likely blue straggler - blue straggler binary system (7782), a SB2 binary with a secondary star which is under-luminous for its mass (5080), two potential eclipsing binaries (4705 and 5762), and two binaries which are likely members of a quadruple system (5015a and 5015b). Finally, in the Appendix we provide the orbital solutions and parameters for the 13 field binaries that we have serendipitously discovered over the course of our survey. The third paper in this series will study the binary frequency of the cluster and analyze the binary distributions in period, eccentricity and secondary mass. With the data analyzed in this series of papers, we will gain a detailed understanding of the cluster dynamics, the properties of the hard-binary population and their influence on the formation of anomalous

\footnotetext{
${ }^{3}$ In the following, as in Geller et al. (2008) (Paper 1) we use the term "single" to identify stars with no significant RV variation; a star is termed single if the standard deviation of its RV measurements is less than four times our precision. Certainly, many of these stars are also binaries, although generally with longer periods and/or lower total mass than the binaries identified in this study.

${ }^{4}$ We adopt a core radius of $1.3 \mathrm{pc}$ (Bonatto, Bica \& Santos Jr. 2005) at a distance of $1.9 \mathrm{kpc}$, which corresponds to 2.35 arcminutes on the sky.
} 
stars like BSs, and thereby provide valuable constraints for future $N$-body models of NGC 188 .

\section{Data}

Our NGC 188 stellar sample spans a magnitude range of $10.8 \leq \mathrm{V} \leq 16.5$ and a $1^{\circ}$ diameter region on the sky. Our magnitude limits include solar-mass main-sequence stars, subgiants, giants, and BSs, and our spatial coverage extends radially to $\sim 13$ core radii. The IDs and coordinates for our stellar sample are taken from the Platais et al. (2003) proper-motion (PM) study. As explained in Paper 1, our full RV database is composed of two data sets, one from WIYN 5 and one from the Dominion Astrophysical Observatory (DAO). The DAO dataset is composed of RVs measured at the DAO $1.2 \mathrm{~m}$ and the Palomar $5 \mathrm{~m}$ telescopes both converted to the DAO Radial-Velocity Spectrometer (RVS) system. Here we present our complete current RV database for each of our observed stars in the field of NGC 188 in Table 11 We include in this table both cluster members and nonmembers as well as stars without sufficient observations to derive membership information. We refer the reader to Paper 1 for thorough descriptions of our stellar sample and its completeness, and where we provide our findings on cluster membership and velocity variability.

We show data for two stars, one SB2 binary and one single star, in Table 1, and provide the full table electronically. For individual RV measurements, we list the reduced Heliocentric Julian Date (HJD-2400000 d), the observatory at which the observations were taken, using "W" for WIYN, "D" for DAO and "P" for Palomar, the measured RV, and the cross-correlation peak height for WIYN measurements as a guide to the quality of measurement (with a maximum value of 1 ; see Paper 1 for a detailed description of the precision of our data as a function of the peak height). For the binaries with orbital solutions, we also provide the residual (O-C), derived as the observed minus the expected RV from the orbital solution, and the phase. For SB2 binaries with orbital solutions, we provide RVs and cross-correlation peak heights (where available) for both stars and their respective residuals.

Observations taken at the WIYN 3.5m range in date from October 1995 through August 2008. Observations made at the DAO 1.2m range in date from February 1980 through November 1996. All observations prior to 1980 were taken at the Palomar 5m, with the earliest observations taken in December 1973. We have found no zero-point offset between the WIYN and DAO data sets (Paper 1), and have thus integrated both sets of measurements without modification into the single RV data set presented here. The precision of the WIYN data is $0.4 \mathrm{~km} \mathrm{~s}^{-1}$ and of the DAO data is $1.0 \mathrm{~km} \mathrm{~s}^{-1}$ (Paper 1).

\section{Spectroscopic Binary Orbits}

In the following section, we present our 85 orbital solutions of the binary members of NGC 188 . We first discuss our 70 SB1 binaries and then our 15 SB2 binaries. For both sets, we provide the tabulated orbital parameters, plotted orbit curves and component mass estimates.

\footnotetext{
${ }^{5}$ The WIYN Observatory is a joint facility of the University of Wisconsin - Madison, Indiana University, Yale University, and the National Optical Astronomy Observatories.
} 
Table 1. Radial-Velocity Data Table

\begin{tabular}{|c|c|c|c|c|c|c|c|c|c|}
\hline ID & $\begin{array}{c}\text { HJD-2400000 } \\
\text { (days) }\end{array}$ & $\mathrm{T}$ & $\begin{array}{c}\mathrm{RV}_{1} \\
\left(\mathrm{~km} \mathrm{~s}^{-1}\right)\end{array}$ & $\begin{array}{c}\text { Correlation } \\
\text { Height }_{1} \\
\end{array}$ & $\begin{array}{c}(\mathrm{O}-\mathrm{C})_{1} \\
\left(\mathrm{~km} \mathrm{~s}^{-1}\right)\end{array}$ & $\begin{array}{c}\mathrm{RV}_{2} \\
\left(\mathrm{~km} \mathrm{~s}^{-1}\right)\end{array}$ & $\begin{array}{c}\text { Correlation } \\
\text { Height }_{2} \\
\end{array}$ & $\begin{array}{c}(\mathrm{O}-\mathrm{C})_{2} \\
\left(\mathrm{~km} \mathrm{~s}^{-1}\right) \\
\end{array}$ & Phase \\
\hline \multicolumn{10}{|l|}{3344} \\
\hline & 50328.945 & $\mathrm{~W}$ & -63.3 & 0.75 & 0.4 & -20.1 & 0.63 & -0.4 & 0.571 \\
\hline & 50331.848 & $\mathrm{~W}$ & -63.2 & 0.43 & 0.5 & -18.7 & 0.32 & 1.0 & 0.594 \\
\hline & 50331.941 & W & -64.6 & 0.51 & -0.9 & -19.1 & 0.39 & 0.6 & 0.595 \\
\hline & 50613.922 & $\mathrm{~W}$ & -44.8 & 0.59 & -0.3 & & & & 0.825 \\
\hline & 50614.746 & $\mathrm{~W}$ & -43.1 & 0.93 & 0.3 & & & & 0.831 \\
\hline & 50615.660 & $\mathrm{~W}$ & -42.6 & 0.56 & -0.5 & & & & 0.838 \\
\hline & 50616.672 & $\mathrm{~W}$ & -41.9 & 0.90 & -1.3 & & & & 0.846 \\
\hline & 50616.895 & W & -42.4 & 0.94 & -2.0 & & & & 0.848 \\
\hline & 50653.902 & $\mathrm{~W}$ & -19.8 & 0.71 & 1.3 & -66.3 & 0.60 & -0.8 & 0.141 \\
\hline & 50703.820 & $\mathrm{~W}$ & -62.9 & 0.66 & 0.2 & -21.2 & 0.53 & -0.9 & 0.535 \\
\hline & 50714.910 & $\mathrm{~W}$ & -63.0 & 0.61 & 0.3 & -19.0 & 0.49 & 1.1 & 0.623 \\
\hline & 50780.637 & $\mathrm{~W}$ & -20.5 & 0.58 & 0.9 & -66.4 & 0.51 & -1.2 & 0.143 \\
\hline & 50797.961 & W & -42.2 & 0.85 & -0.3 & & & & 0.280 \\
\hline & 50815.594 & $\mathrm{~W}$ & -56.9 & 0.74 & 0.2 & -30.5 & 0.67 & -3.8 & 0.419 \\
\hline & 50918.957 & $\mathrm{~W}$ & -35.5 & 0.49 & 0.1 & -47.4 & 0.48 & 2.4 & 0.237 \\
\hline & 50920.941 & $\mathrm{~W}$ & -37.8 & 0.55 & 0.2 & -48.7 & 0.52 & -1.3 & 0.252 \\
\hline & 50921.941 & W & -40.2 & 0.41 & -1.0 & -47.4 & 0.40 & -1.3 & 0.260 \\
\hline & 50976.676 & $\mathrm{~W}$ & -59.3 & 0.62 & 0.8 & -23.9 & 0.53 & -0.4 & 0.693 \\
\hline & 50996.750 & $\mathrm{~W}$ & -40.3 & 0.84 & -0.7 & -46.5 & 0.82 & -0.9 & 0.852 \\
\hline & 51127.672 & $\mathrm{~W}$ & -32.2 & 0.78 & 0.7 & -54.5 & 0.70 & -1.6 & 0.887 \\
\hline & 51128.648 & $\mathrm{~W}$ & -28.6 & 0.83 & 2.8 & -54.3 & 0.73 & 0.2 & 0.895 \\
\hline & 51174.980 & W & -40.3 & 0.89 & -1.0 & -46.8 & 0.85 & -0.8 & 0.261 \\
\hline & 51176.012 & W & -42.1 & 0.93 & -1.6 & & & & 0.269 \\
\hline & 51179.039 & $\mathrm{~W}$ & -41.6 & 0.77 & 2.2 & & & & 0.293 \\
\hline & 51186.973 & $\mathrm{~W}$ & -50.8 & 0.75 & 0.5 & -33.4 & 0.70 & -0.3 & 0.356 \\
\hline & 51243.863 & $\mathrm{~W}$ & -45.0 & 0.67 & 2.5 & -39.3 & 0.66 & -2.2 & 0.806 \\
\hline & 51355.922 & $\mathrm{~W}$ & -58.6 & 0.80 & 1.5 & -25.4 & 0.68 & -1.9 & 0.692 \\
\hline & 53389.984 & $\mathrm{~W}$ & -52.3 & 0.52 & -0.6 & -29.8 & 0.61 & 2.8 & 0.777 \\
\hline \multicolumn{10}{|l|}{3359} \\
\hline & 50614.777 & $\mathrm{~W}$ & -42.7 & 0.91 & & & & & \\
\hline & 50616.922 & $\mathrm{~W}$ & -42.9 & 0.91 & & & & & \\
\hline & 50653.930 & W & -42.6 & 0.89 & & & & & \\
\hline & 50703.836 & $\mathrm{~W}$ & -42.2 & 0.86 & & & & & \\
\hline & 50714.953 & W & -43.0 & 0.67 & & & & & \\
\hline & 50780.648 & $\mathrm{~W}$ & -42.5 & 0.85 & & & & & \\
\hline & 50797.992 & $\mathrm{~W}$ & -42.2 & 0.88 & & & & & \\
\hline & 50815.625 & W & -41.8 & 0.92 & & & & & \\
\hline & 50857.012 & W & -42.0 & 0.75 & & & & & \\
\hline & 50918.977 & $\mathrm{~W}$ & -42.2 & 0.63 & & & & & \\
\hline & 50920.961 & $\mathrm{~W}$ & -41.5 & 0.83 & & & & & \\
\hline & 50976.695 & $\mathrm{~W}$ & -42.3 & 0.77 & & & & & \\
\hline & 50996.777 & W & -42.5 & 0.77 & & & & & \\
\hline & 51498.625 & W & -42.2 & 0.95 & & & & & \\
\hline & 51921.875 & $\mathrm{~W}$ & -42.3 & 0.85 & & & & & \\
\hline & 52462.945 & W & -42.0 & 0.92 & & & & & \\
\hline & 53074.992 & $\mathrm{~W}$ & -43.0 & 0.75 & & & & & \\
\hline
\end{tabular}

(This table is available in its entirety in a machine-readable form in the online journal. A portion is shown here for guidance regarding its form and content) 


\subsection{Single-Lined Orbital Solutions}

For each SB1 binary, we solve for the orbital solution using the data given in Table 1, We provide the plotted orbital solutions in Figure 1; for each binary we plot the orbit in the top panel and the RV residuals in the bottom panel. In Table 2 we provide the orbital elements for each binary in two rows, where the first row includes the binary ID, the orbital period $(P)$, the number of orbital cycles observed, the center-of-mass RV $(\gamma)$, the orbital amplitude $(K)$, the eccentricity $(e)$, the longitude of periastron $(\omega)$, a Julian Date of periastron passage $\left(T_{\circ}\right)$, the projected semi-major axis $(a \sin i)$, the mass function $(f(m))$, the rms residual velocity from the orbital solution $(\sigma)$, and the number of RV measurements $(N)$. Where applicable, the second row contains the respective errors on each of these values. In Table 3, we present physical properties for each SB1, including the WOCS ID, the $V$ magnitude and the $(B-V)$ color (both from Stetson et al. 2004), the radial distance from the cluster center (in arcminutes), the RV membership probability $\left(P_{R V}\right.$; from Paper 1), the PM membership probability $\left(P_{P M}\right.$; from Platais et al. 2003), a photometric estimate for the mass of the primary $\left(M_{1}\right)$, a lower limit for the mass of the secondary $\left(M_{2} \min \right)$, and finally a photometric estimate for the mass of the secondary $\left(M_{2}\right)$.

The photometric estimates for the primary and secondary masses are derived simultaneously across the available $U B V R I$ photometry for each binary using a photometric deconvolution technique. We use the observed $(U-V),(B-V),(V-R)$, and $(V-I)$ colors, where available, and $V$ magnitudes (as compiled by Stetson et al. (2004)) along with a 7 Gyr, solar-metallicity Padova isochrone6 (Girardi et al. 2002) to produce a set of synthetic binaries. This set of binaries contains primary stars within a range of masses whose magnitudes extend from the observed $V$ magnitude to this magnitude plus 0.75 (as this would be the contribution from an equal mass companion) and, for each primary star, a set of secondary stars of equal or lesser mass. The component masses of the synthetic binary that has a composite $V$ magnitude and colors in the available photometric bands that most closely match the observed $V$ magnitude and colors, in both color-magnitude and color-color space, are taken as the photometric primary and secondary mass estimates.

We only attempt to quote masses for the main-sequence, sub-giant and giant binaries. We caution the reader that, for binaries with mass ratios $\lesssim 0.5$, the photometric masses are less certain, as solar-type binaries with these low mass ratios fall very near to the isochrone (e.g., Hurley \& Tout 1998). Also the morphology of the isochrone near the turnoff, makes the masses for binaries in this region more sensitive to selection of the distance modulus. In certain cases (e.g., when the observed binary lies directly on the isochrone to within the photometric errors, or the binary is found blueward of the main-sequence or redward of the giant branch), we cannot derive reliable mass estimates in the manner described above. For such cases, we use the observed $V$ magnitude to estimate an upper limit on the mass of the primary star. We have found that the secondary must be at least 2.5 magnitudes fainter than the primary at a central wavelength of $5250 \AA$ (the central wavelength of the WIYN spectra) for the binary to be observed as single lined. Thus, in these cases, we use this resulting upper limit on the $V$ magnitude for the secondary to derive the upper limit on its mass (and note this in the table). Finally, for all SB1 binaries we use the primary mass estimate along with the orbital mass function to derive a lower limit on the secondary mass.

For two binaries, 4965 and 4688, we notice a clear trend with time in the residuals of the orbital solutions fit to the observed RVs. We assume that this trend is due to the presence of an additional longperiod companion (or companions). Therefore, for each of these two binaries, we fit a polynomial function (of first and second order, respectively) to the residuals, subtract this fit from the observed RVs, and refit

\footnotetext{
${ }^{6}$ For the isochrone, we set $E(B-V)=0.025$ and $(m-M)_{V}=11.23$ (Fornal et al. 2007).
} 
the orbit to these corrected RVs. There is no trend in the resulting residuals from the corrected orbital solutions for either of these binaries. We note that all of the orbital parameters derived from the corrected orbital solutions agree with those of the uncorrected orbital solutions to within the errors, except for two parameters in 4688; the orbital amplitude, $K$, increased from $6.7 \pm 0.5$ in the uncorrected orbit to $9.6 \pm 1.7$ in the corrected orbit, and the orbital eccentricity, $e$, increased from $0.57 \pm 0.05$ in the uncorrected orbit to $0.70 \pm 0.04$ in the corrected orbit. We show the corrected orbital solution plots in Figure 1 and parameters in Table 2. In our RV data table, Table 1, we include the observed RVs and the residuals to the corrected orbital solutions.

Curiously, this SB1 photometric deconvolution technique has yielded three cases where we would expect to see the secondary. Binaries 4524 and 4843 lie well blueward of the giant branch, and binary 4390 lies well redward of the main sequence. We also note that some spectra of 4710 reveal an additionally component for which we have no current explanation. This binary is located near the main-sequence turnoff. The rest of the mass estimates yield luminosity ratios in which we indeed would not expect to observe the secondary star, given our observing setup.

We use a Monte Carlo technique to estimate the mean uncertainty on our mass estimates, assuming this uncertainty to be derived from two main sources: the uncertainties on the photometry and on the isochrone fit. For binaries in which we can estimate masses from the photometric deconvolution technique, we find a mean uncertainty for the primary mass of $0.09 \mathrm{M}_{\odot}$ and on the secondary of $0.14 \mathrm{M}_{\odot}$. The standard deviations about these means are $0.15 \mathrm{M}_{\odot}$ and $0.20 \mathrm{M}_{\odot}$, respectively. Uncertainties on the minimum secondary masses are found in a similar manner, using the derived primary mass uncertainty along with the error on the mass function resulting from the orbital solution, and result in a mean uncertainty of $0.04 \mathrm{M}_{\odot}$ with a standard deviation about the mean of $0.10 \mathrm{M}_{\odot}$. Finally, for binaries in which we can only give limits on the primary and secondary masses, we note that the mean uncertainty on the $V$ magnitudes for all binaries is 0.011 magnitudes. For solar-type stars, a shift of this amount to the observed magnitude of a main-sequence star results in a shift in mass of $0.003 \mathrm{M}_{\odot}$.
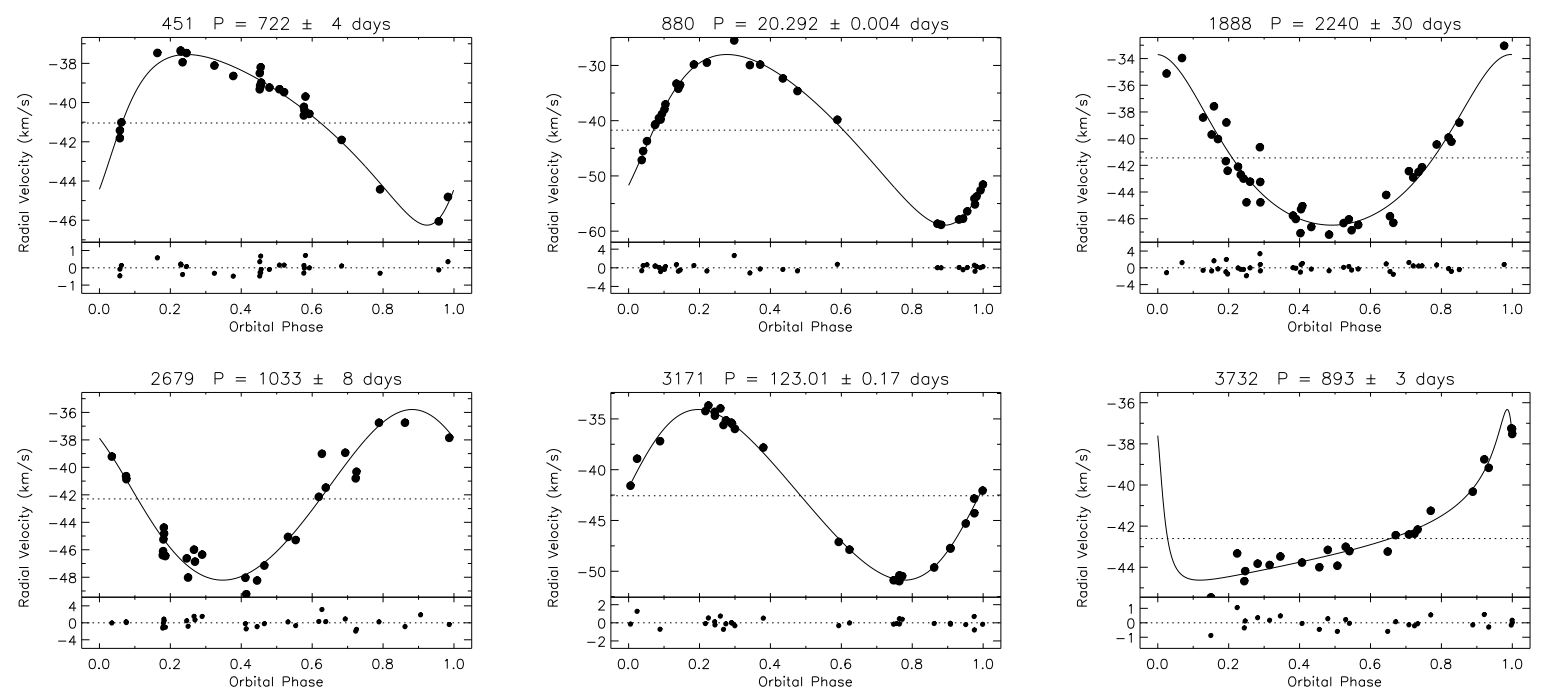

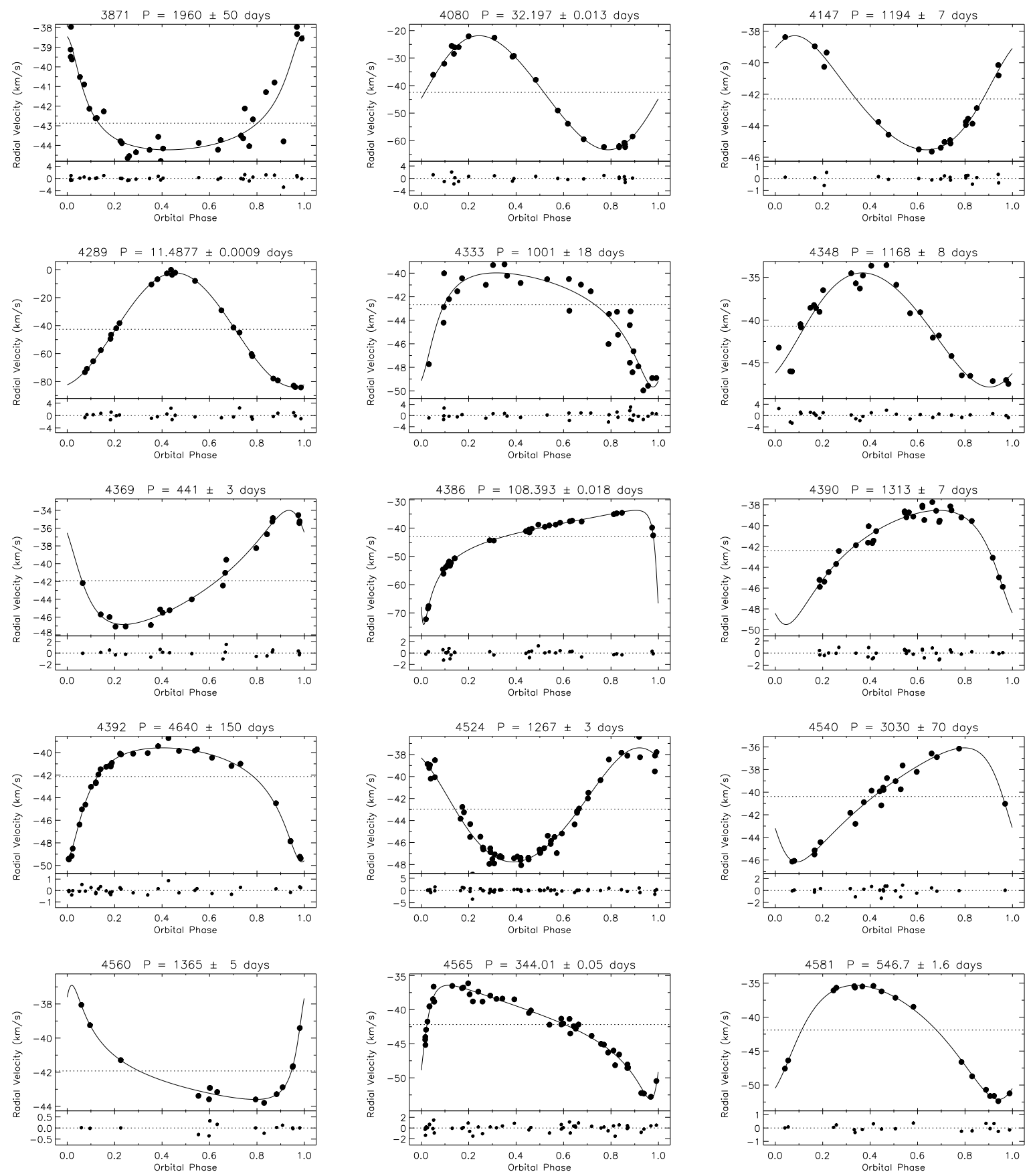

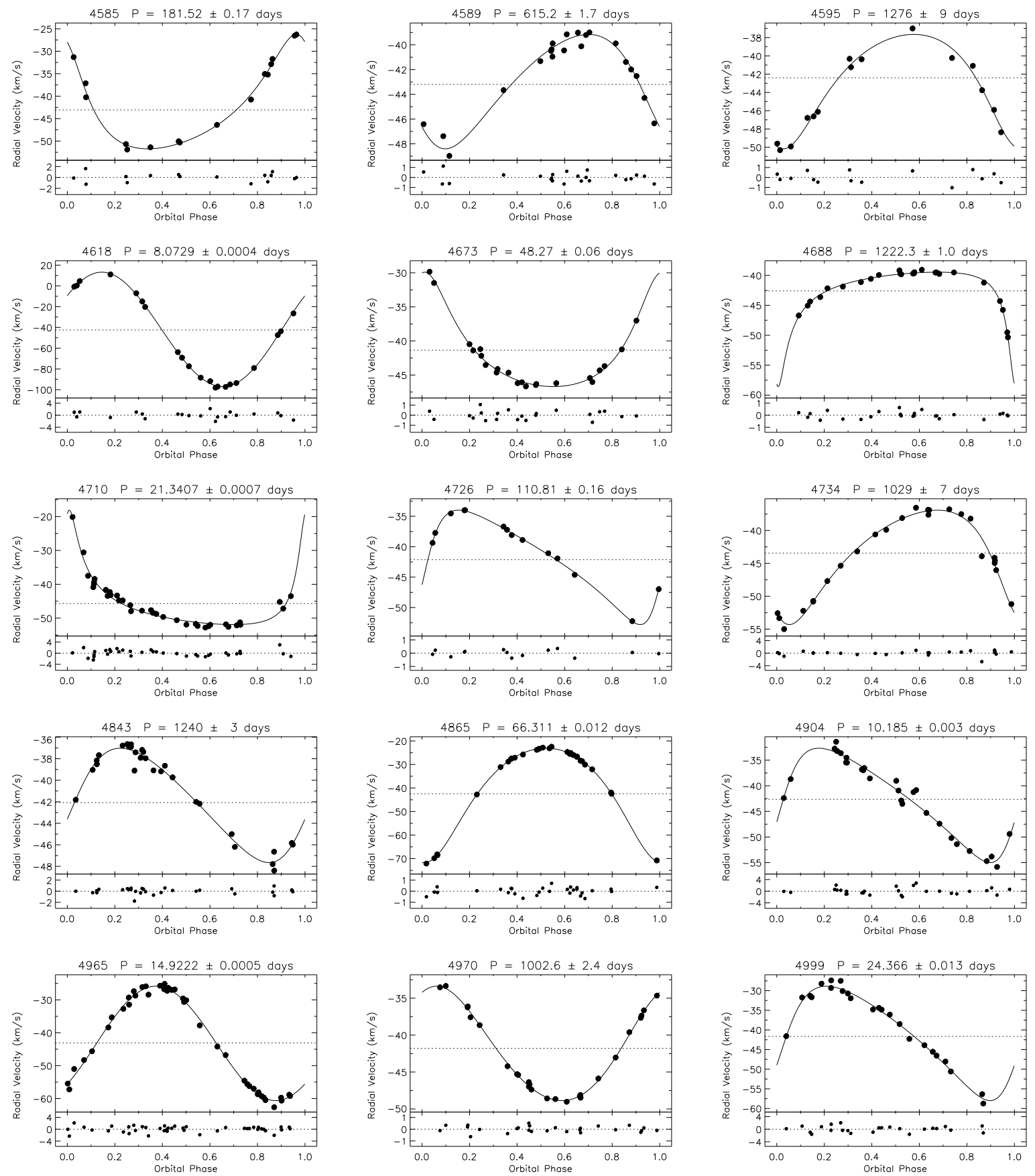

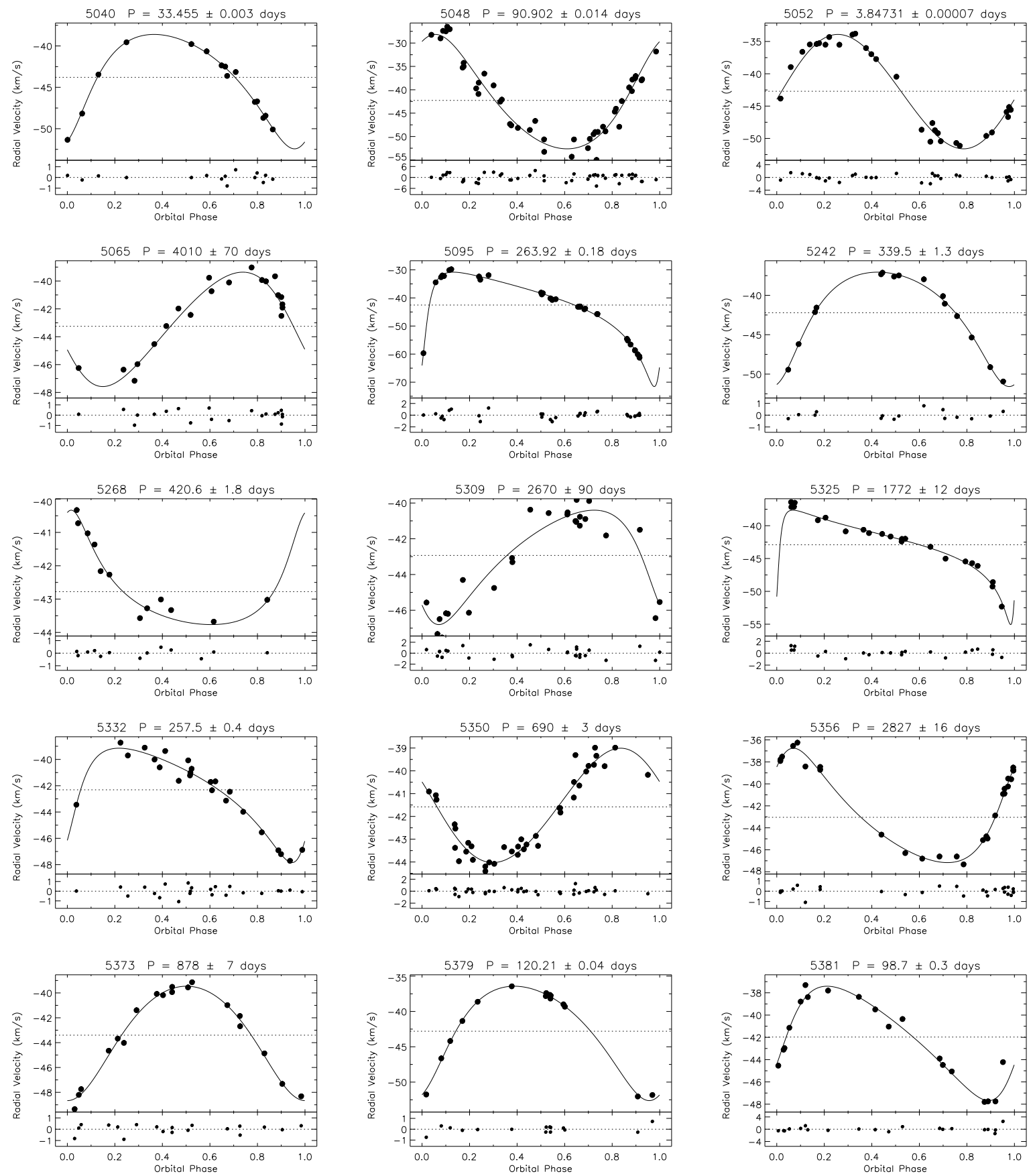

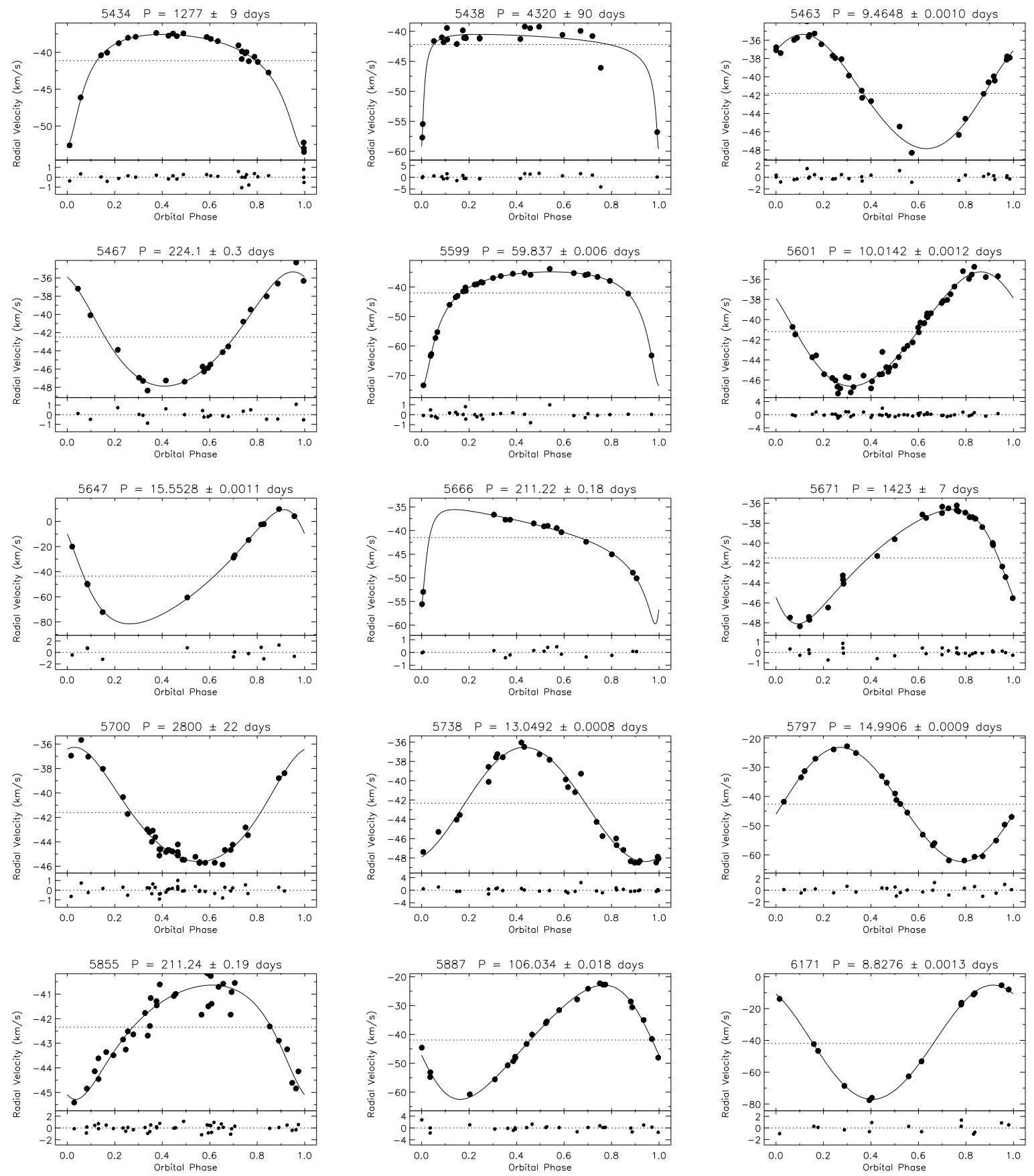

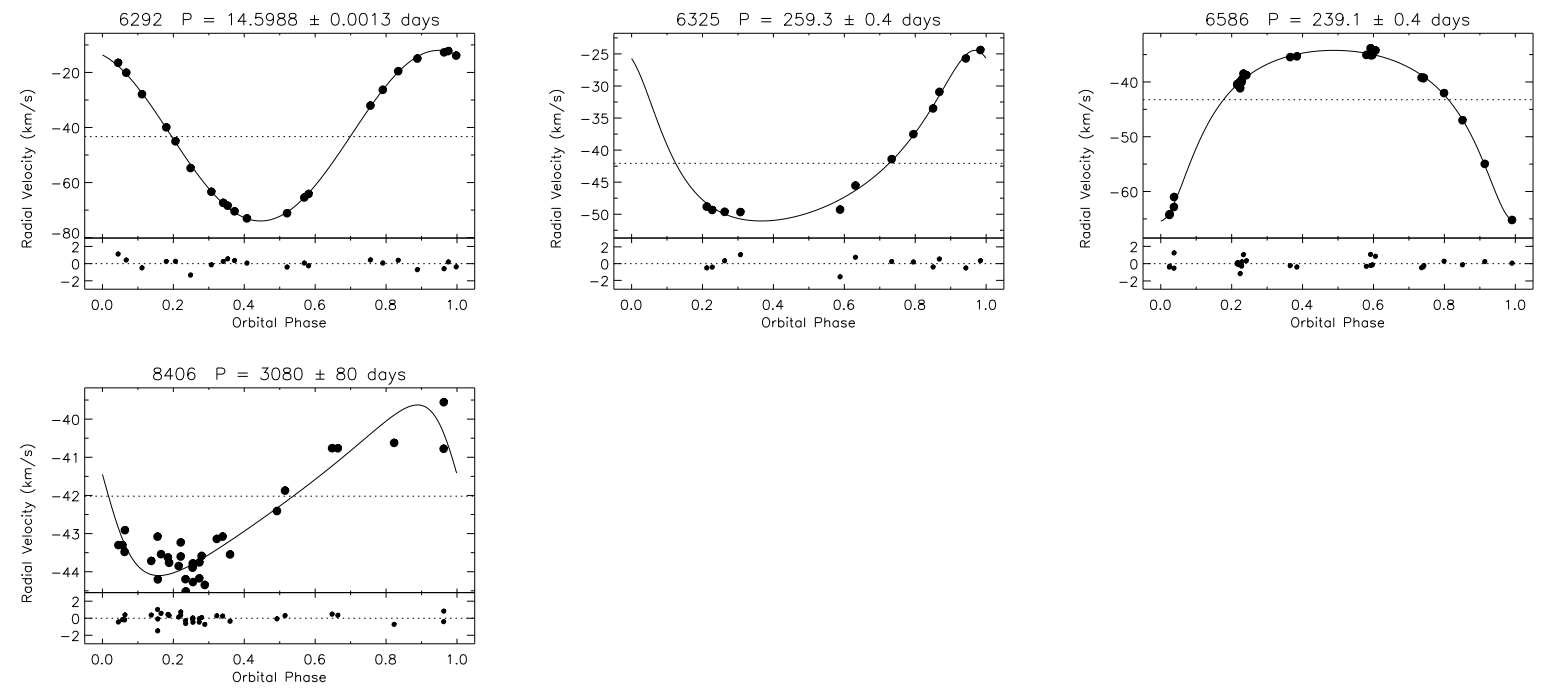

Fig. 1. - NGC 188 SB1 orbit plots. For each binary, we plot RV against orbital phase, showing the data points with black dots and the orbital fit to the data with the solid line; the dotted line marks the $\gamma$-velocity. Beneath each orbit plot, we show the residuals from the fit. Above each plot, we give the binary ID and orbital period. 
Table 2. Orbital Parameters For NGC 188 Single-Lined Binaries

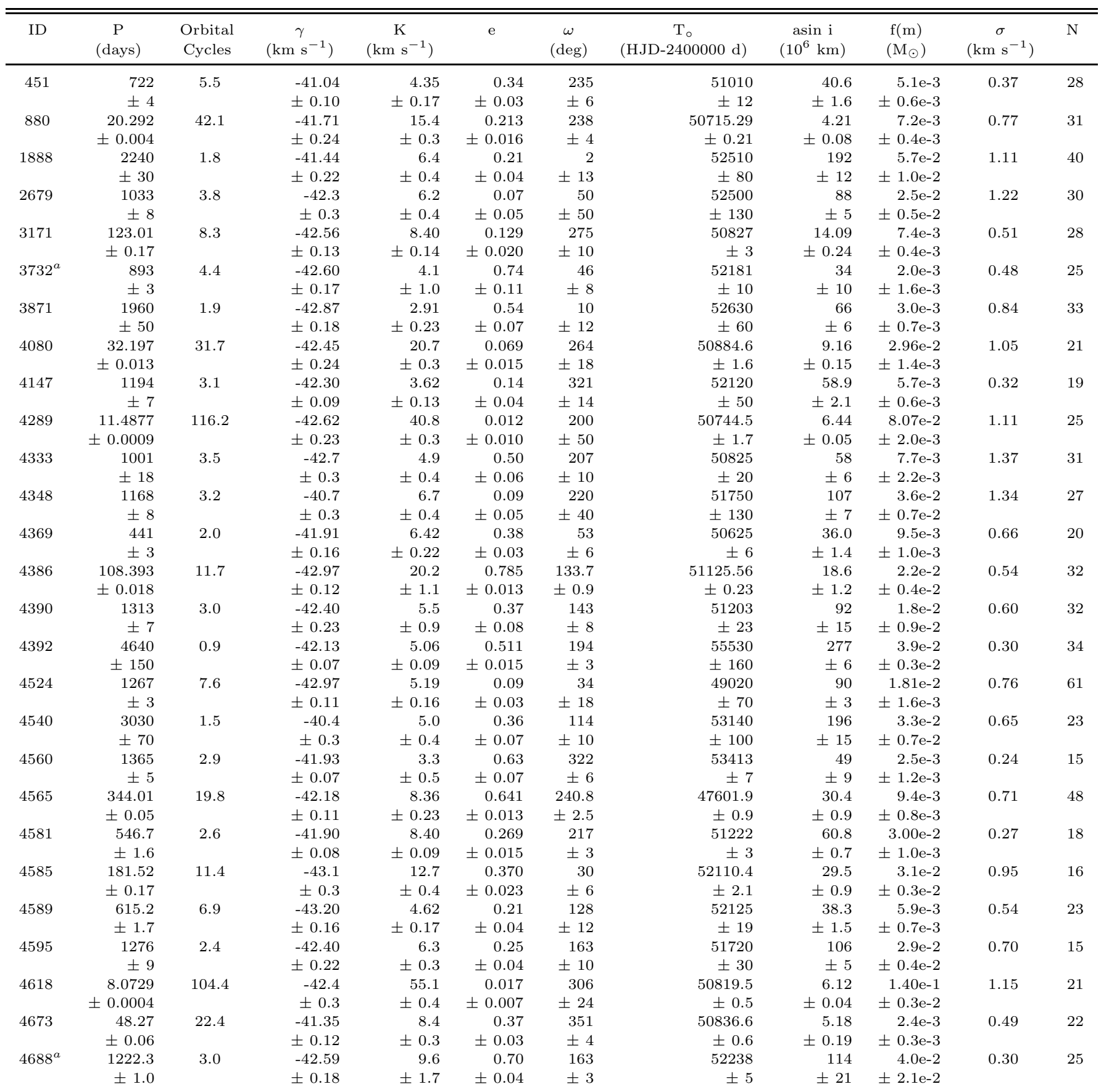


Table 2-Continued

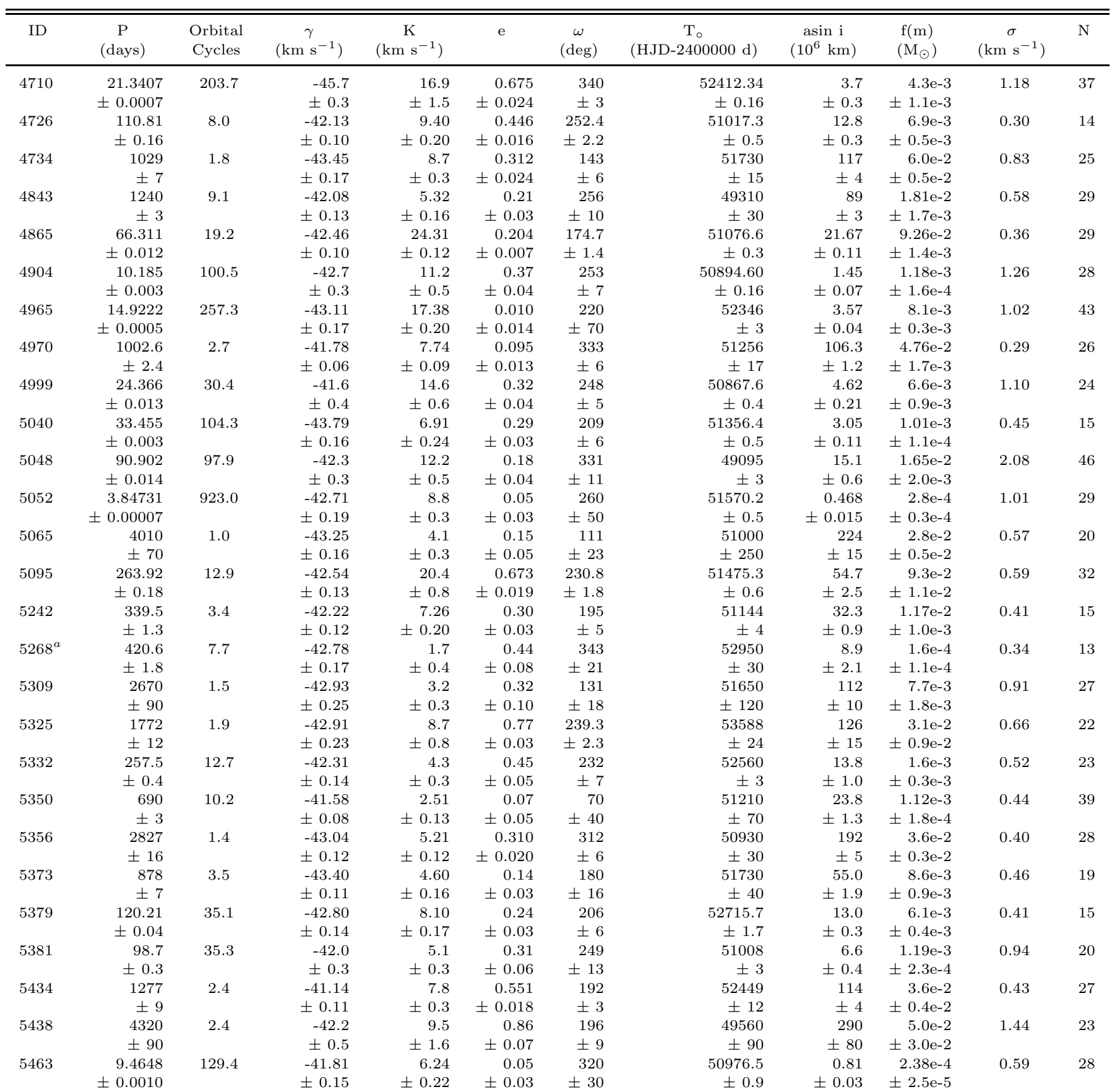


Table 2-Continued

\begin{tabular}{|c|c|c|c|c|c|c|c|c|c|c|c|}
\hline ID & $\begin{array}{c}\mathrm{P} \\
\text { (days) }\end{array}$ & $\begin{array}{l}\text { Orbital } \\
\text { Cycles }\end{array}$ & $\left(\mathrm{km} \mathrm{s}^{-1}\right)$ & $\begin{array}{c}\mathrm{K} \\
\left(\mathrm{km} \mathrm{s}^{-1}\right)\end{array}$ & e & $\begin{array}{c}\omega \\
(\mathrm{deg})\end{array}$ & $\begin{array}{c}\mathrm{T}_{\circ} \\
(\mathrm{HJD}-2400000 \mathrm{~d})\end{array}$ & $\begin{array}{c}\operatorname{asin} \mathrm{i} \\
\left(10^{6} \mathrm{~km}\right)\end{array}$ & $\begin{array}{l}\mathrm{f}(\mathrm{m}) \\
\left(\mathrm{M}_{\odot}\right)\end{array}$ & $\left(\begin{array}{c}\sigma \\
\left(\mathrm{km} \mathrm{s}^{-1}\right)\end{array}\right.$ & $\mathrm{N}$ \\
\hline 5467 & $\begin{array}{l}224.1 \\
\pm 0.3\end{array}$ & 11.0 & $\begin{array}{l}-42.48 \\
\pm 0.14\end{array}$ & $\begin{array}{r}6.27 \\
\pm 0.19\end{array}$ & $\begin{array}{r}0.15 \\
\pm 0.03\end{array}$ & $\begin{array}{r}25 \\
\pm 10\end{array}$ & $\begin{array}{r}51153 \\
\pm 6\end{array}$ & $\begin{array}{r}19.1 \\
\pm 0.6\end{array}$ & $\begin{array}{r}5.5 \mathrm{e}-3 \\
\pm 0.5 \mathrm{e}-3\end{array}$ & 0.57 & 20 \\
\hline 5599 & $\begin{array}{r}59.837 \\
\pm 0.006\end{array}$ & 32.5 & $\begin{array}{l}-42.01 \\
\pm 0.10\end{array}$ & $\begin{array}{r}19.38 \\
\pm 0.22\end{array}$ & $\begin{array}{r}0.633 \\
\pm 0.005\end{array}$ & $\begin{array}{l}175.4 \\
\pm 0.6\end{array}$ & $\begin{array}{r}51879.10 \\
\pm 0.09\end{array}$ & $\begin{array}{r}12.34 \\
\pm 0.16\end{array}$ & $\begin{array}{r}2.09 \mathrm{e}-2 \\
\pm 0.8 \mathrm{e}-3\end{array}$ & 0.39 & 28 \\
\hline 5601 & $\begin{array}{r}10.0142 \\
\pm 0.0012\end{array}$ & 132.6 & $\begin{array}{r}-41.19 \\
\pm 0.10\end{array}$ & $\begin{array}{r}5.67 \\
\pm 0.10\end{array}$ & $\begin{array}{r}0.09 \\
\pm 0.03\end{array}$ & $\begin{array}{r}58 \\
\pm 16\end{array}$ & $\begin{array}{r}51162.9 \\
\pm 0.4\end{array}$ & $\begin{array}{r}0.779 \\
\pm 0.020\end{array}$ & $\begin{array}{r}1.87 \mathrm{e}-4 \\
\pm 1.4 \mathrm{e}-5\end{array}$ & 0.57 & 47 \\
\hline 5647 & $\begin{array}{r}15.5528 \\
\pm 0.0011\end{array}$ & 57.3 & $\begin{array}{l}-43.5 \\
\pm 0.6\end{array}$ & $\begin{array}{r}45.5 \\
\pm 0.7\end{array}$ & $\begin{array}{r}0.285 \\
\pm 0.011\end{array}$ & $\begin{array}{r}55 \\
\pm 3\end{array}$ & $\begin{array}{r}54123.82 \\
\pm 0.10\end{array}$ & $\begin{array}{r}9.32 \\
\pm 0.15\end{array}$ & $\begin{array}{r}1.33 \mathrm{e}-1 \\
\pm 0.6 \mathrm{e}-2\end{array}$ & 1.17 & 12 \\
\hline 5666 & $\begin{array}{l}211.22 \\
\pm 0.18\end{array}$ & 13.3 & $\begin{array}{l}-41.5 \\
\pm 0.3\end{array}$ & $\begin{array}{r}12.1 \\
\pm 0.6\end{array}$ & $\begin{array}{r}0.712 \\
\pm 0.019\end{array}$ & $\begin{array}{l}224 \\
\pm 5\end{array}$ & $\begin{array}{r}53321.7 \\
\pm 0.7\end{array}$ & $\begin{array}{r}24.7 \\
\pm 1.5\end{array}$ & $\begin{array}{r}1.34 \mathrm{e}-2 \\
\pm 2.2 \mathrm{e}-3\end{array}$ & 0.32 & 14 \\
\hline 5671 & $\begin{array}{r}1423 \\
\pm 7\end{array}$ & 2.1 & $\begin{array}{r}-41.49 \\
\pm 0.08\end{array}$ & $\begin{array}{r}5.74 \\
\pm 0.10\end{array}$ & $\begin{array}{r}0.286 \\
\pm 0.018\end{array}$ & $\begin{array}{l}123 \\
\pm 4\end{array}$ & $\begin{array}{r}51044 \\
\pm 12\end{array}$ & $\begin{array}{l}107.6 \\
\pm 2.0\end{array}$ & $\begin{array}{r}2.45 \mathrm{e}-2 \\
\pm 1.3 \mathrm{e}-3\end{array}$ & 0.35 & 30 \\
\hline 5700 & $\begin{array}{l}2800 \\
\pm 22\end{array}$ & 3.7 & $\begin{array}{r}-41.61 \\
\pm 0.11\end{array}$ & $\begin{array}{r}4.68 \\
\pm 0.13\end{array}$ & $\begin{array}{r}0.15 \\
\pm 0.03\end{array}$ & $\begin{array}{r}345 \\
\pm 10\end{array}$ & $\begin{array}{r}52420 \\
\pm 80\end{array}$ & $\begin{array}{l}178 \\
\pm 5\end{array}$ & $\begin{array}{r}2.88 \mathrm{e}-2 \\
\pm 2.5 \mathrm{e}-3\end{array}$ & 0.44 & 38 \\
\hline 5738 & $\begin{array}{r}13.0492 \\
\pm 0.0008\end{array}$ & 272.3 & $\begin{array}{l}-42.33 \\
\pm 0.15\end{array}$ & $\begin{array}{r}5.92 \\
\pm 0.20\end{array}$ & $\begin{array}{r}0.02 \\
\pm 0.04\end{array}$ & $\begin{array}{r}200 \\
\pm 80\end{array}$ & $\begin{array}{r}51403 \\
\pm 3\end{array}$ & $\begin{array}{r}1.06 \\
\pm 0.04\end{array}$ & $\begin{array}{r}2.8 \mathrm{e}-4 \\
\pm 0.3 \mathrm{e}-4\end{array}$ & 0.74 & 29 \\
\hline 5797 & $\begin{array}{r}14.9906 \\
\pm 0.0009\end{array}$ & 98.8 & $\begin{array}{l}-42.67 \\
\pm 0.15\end{array}$ & $\begin{array}{r}19.53 \\
\pm 0.23\end{array}$ & $\begin{array}{r}0.023 \\
\pm 0.011\end{array}$ & $\begin{array}{r}260 \\
\pm 30\end{array}$ & $\begin{array}{r}52033.9 \\
\pm 1.1\end{array}$ & $\begin{array}{r}4.02 \\
\pm 0.05\end{array}$ & $\begin{array}{r}1.16 \mathrm{e}-2 \\
\pm 0.4 \mathrm{e}-3\end{array}$ & 0.69 & 23 \\
\hline 5855 & $\begin{array}{r}211.24 \\
\pm 0.19\end{array}$ & 47.0 & $\begin{array}{l}-42.35 \\
\pm 0.10\end{array}$ & $\begin{array}{r}2.32 \\
\pm 0.18\end{array}$ & $\begin{array}{r}0.29 \\
\pm 0.07\end{array}$ & $\begin{array}{r}157 \\
\pm 12\end{array}$ & $\begin{array}{r}50203 \\
\pm 6\end{array}$ & $\begin{array}{r}6.5 \\
\pm 0.5\end{array}$ & $\begin{array}{r}2.4 \mathrm{e}-4 \\
\pm \quad 0.6 \mathrm{e}-4\end{array}$ & 0.59 & 41 \\
\hline 5887 & $\begin{array}{l}106.034 \\
\pm 0.018\end{array}$ & 57.5 & $\begin{array}{l}-41.9 \\
\pm 0.3\end{array}$ & $\begin{array}{r}19.8 \\
\pm 0.5\end{array}$ & $\begin{array}{r}0.171 \\
\pm 0.018\end{array}$ & $\begin{array}{l}103 \\
\pm 6\end{array}$ & $\begin{array}{r}55583.1 \\
\pm 1.8\end{array}$ & $\begin{array}{r}28.5 \\
\pm 0.7\end{array}$ & $\begin{array}{r}8.2 \mathrm{e}-2 \\
\pm 0.6 \mathrm{e}-2\end{array}$ & 1.09 & 24 \\
\hline 6171 & $\begin{array}{r}8.8276 \\
\pm 0.0013\end{array}$ & 125.4 & $\begin{array}{l}-41.9 \\
\pm 0.3\end{array}$ & $\begin{array}{r}35.9 \\
\pm \quad 0.4\end{array}$ & $\begin{array}{r}0.025 \\
\pm 0.011\end{array}$ & $\begin{array}{r}33 \\
\pm 24\end{array}$ & $\begin{array}{r}54189.8 \\
\pm 0.6\end{array}$ & $\begin{array}{r}4.36 \\
\pm 0.05\end{array}$ & $\begin{array}{r}4.24 \mathrm{e}-2 \\
\pm 1.3 \mathrm{e}-3\end{array}$ & 0.97 & 14 \\
\hline 6292 & $\begin{array}{r}14.5988 \\
\pm 0.0013\end{array}$ & 70.1 & $\begin{array}{r}-43.31 \\
\pm 0.14\end{array}$ & $\begin{array}{r}30.99 \\
\pm 0.19\end{array}$ & $\begin{array}{r}0.012 \\
\pm 0.007\end{array}$ & $\begin{array}{r}20 \\
\pm 30\end{array}$ & $\begin{array}{r}50859.9 \\
\pm 1.3\end{array}$ & $\begin{array}{r}6.22 \\
\pm 0.04\end{array}$ & $\begin{array}{r}4.50 \mathrm{e}-2 \\
\pm 0.8 \mathrm{e}-3\end{array}$ & 0.62 & 21 \\
\hline $6325^{a}$ & $\begin{array}{r}259.3 \\
\pm 0.4\end{array}$ & 11.8 & $\begin{array}{r}-42.1 \\
\pm 0.4\end{array}$ & $\begin{array}{r}13.3 \\
\pm 0.5\end{array}$ & $\begin{array}{r}0.36 \\
\pm 0.05\end{array}$ & $\begin{array}{r}25 \\
\pm 6\end{array}$ & $\begin{array}{r}52995 \\
\pm 6\end{array}$ & $\begin{array}{r}44.3 \\
\pm 1.8\end{array}$ & $\begin{array}{r}5.1 \mathrm{e}-2 \\
\pm 0.6 \mathrm{e}-2\end{array}$ & 0.98 & 12 \\
\hline 6586 & $\begin{array}{l}239.1 \\
\pm 0.4\end{array}$ & 5.1 & $\begin{array}{r}-43.24 \\
\pm 0.12\end{array}$ & $\begin{array}{r}15.58 \\
\pm 0.22\end{array}$ & $\begin{array}{r}0.422 \\
\pm 0.013\end{array}$ & $\begin{array}{l}181.9 \\
\pm 1.5\end{array}$ & $\begin{array}{r}50562.2 \\
\pm 1.0\end{array}$ & $\begin{array}{r}46.5 \\
\pm 0.7\end{array}$ & $\begin{array}{r}7.0 \mathrm{e}-2 \\
\pm 0.3 \mathrm{e}-2\end{array}$ & 0.57 & 29 \\
\hline 8406 & $\begin{array}{l}3080 \\
\pm 80\end{array}$ & 1.3 & $\begin{array}{r}-42.02 \\
\pm 0.15\end{array}$ & $\begin{array}{r}2.24 \\
\pm 0.24\end{array}$ & $\begin{array}{r}0.38 \\
\pm 0.06\end{array}$ & $\begin{array}{r}79 \\
\pm 16\end{array}$ & $\begin{array}{l}53210 \\
\pm 120\end{array}$ & $\begin{array}{r}88 \\
+10\end{array}$ & $\begin{array}{r}2.8 \mathrm{e}-3 \\
\pm 0.9 \mathrm{e}-3\end{array}$ & 0.56 & 34 \\
\hline
\end{tabular}

${ }^{a}$ We caution the reader that, though these orbital solutions appear to be robust, these binaries have poor phase coverage. 
Table 3. Physical Properties of NGC 188 Single-Lined Binaries

\begin{tabular}{|c|c|c|c|c|c|c|c|c|}
\hline ID & $V$ & $(B-V)$ & $\begin{array}{c}\mathrm{R} \\
(\operatorname{arcmin})\end{array}$ & $\begin{array}{l}\mathrm{P}_{R V} \\
(\%)\end{array}$ & $\begin{array}{c}\mathrm{P}_{P M} \\
(\%)\end{array}$ & $\begin{array}{c}\mathrm{M}_{1} \\
\left(\mathrm{M}_{\odot}\right)\end{array}$ & $\begin{array}{c}\mathrm{M}_{2} \min \\
\left(\mathrm{M}_{\odot}\right)\end{array}$ & $\begin{array}{c}\mathrm{M}_{2} \\
\left(\mathrm{M}_{\odot}\right)\end{array}$ \\
\hline 880 & 15.383 & 0.684 & 16.67 & 97 & 98 & 1.04 & 0.23 & 0.69 \\
\hline 3171 & 15.077 & 0.911 & 15.79 & 98 & 98 & $<1.12$ & 0.24 & $<0.80$ \\
\hline 3732 & 14.935 & 0.687 & 11.89 & 98 & 96 & 1.08 & 0.15 & 0.75 \\
\hline 3871 & 15.300 & 0.648 & 12.78 & 97 & 89 & 1.05 & 0.16 & 0.45 \\
\hline 4080 & 15.524 & 0.674 & 7.76 & 98 & 98 & 1.03 & 0.39 & 0.60 \\
\hline 4147 & 15.161 & 0.649 & 13.56 & 98 & 98 & $<1.07$ & 0.21 & $<0.79$ \\
\hline 4289 & 15.303 & 0.937 & 5.97 & 98 & 98 & $<1.12$ & 0.58 & $<0.78$ \\
\hline 4333 & 15.857 & 0.676 & 6.11 & 98 & 98 & $<1.00$ & 0.23 & $<0.72$ \\
\hline 4369 & 15.549 & 0.660 & 6.61 & 98 & 98 & $<1.03$ & 0.25 & $<0.75$ \\
\hline 4386 & 15.442 & 0.660 & 7.03 & 98 & 63 & 1.04 & 0.35 & 0.55 \\
\hline 4390 & 15.662 & 0.741 & 6.52 & 98 & 97 & 0.99 & 0.31 & 0.80 \\
\hline 4392 & 15.191 & 0.647 & 6.01 & 98 & 97 & 1.04 & 0.44 & 0.55 \\
\hline 4524 & 12.434 & 1.165 & 5.44 & 98 & 97 & 1.14 & 0.34 & 1.14 \\
\hline 4560 & 15.061 & 0.667 & 3.78 & 97 & 98 & 1.08 & 0.16 & 0.33 \\
\hline 4565 & 12.416 & 1.273 & 3.72 & 98 & 96 & $<1.14$ & 0.27 & $<1.10$ \\
\hline 4585 & 15.814 & 0.666 & 4.41 & 98 & 98 & $<1.00$ & 0.40 & $<0.73$ \\
\hline 4595 & 15.055 & 0.660 & 2.56 & 98 & 97 & $<1.08$ & 0.40 & $<0.80$ \\
\hline 4618 & 15.772 & 0.712 & 3.10 & 98 & 98 & 1.00 & 0.75 & 0.75 \\
\hline 4673 & 14.880 & 0.716 & 2.06 & 95 & 98 & 1.11 & 0.16 & 0.64 \\
\hline 4688 & 15.079 & 0.636 & 3.33 & 98 & 98 & $<1.07$ & 0.45 & $<0.80$ \\
\hline 4710 & 14.846 & 0.667 & 2.89 & 1 & 96 & 1.09 & 0.19 & 0.60 \\
\hline 4726 & 15.302 & 0.671 & 4.46 & 98 & 98 & $<1.05$ & 0.22 & $<0.78$ \\
\hline 4734 & 14.950 & 0.997 & 4.74 & 96 & 98 & 1.13 & 0.55 & 0.64 \\
\hline 4843 & 11.541 & 1.302 & 8.77 & 98 & 75 & 1.14 & 0.34 & 1.14 \\
\hline 4865 & 14.930 & 0.783 & 7.64 & 98 & 95 & $<1.12$ & 0.66 & $<0.81$ \\
\hline 4904 & 15.930 & 0.733 & 5.48 & 98 & 96 & 0.97 & 0.11 & 0.60 \\
\hline 4965 & 15.282 & 0.693 & 2.36 & 96 & 85 & 1.03 & 0.24 & 0.71 \\
\hline 4999 & 15.792 & 0.664 & 0.99 & 97 & 97 & $<1.00$ & 0.22 & $<0.73$ \\
\hline 5040 & 15.009 & 0.667 & 0.68 & 92 & 97 & 1.09 & 0.11 & 0.49 \\
\hline 5048 & 13.869 & 1.069 & 0.84 & 98 & 94 & 1.14 & 0.33 & 0.74 \\
\hline 5052 & 15.916 & 0.710 & 0.33 & 98 & 98 & 0.99 & 0.07 & 0.62 \\
\hline 5065 & 15.754 & 0.746 & 1.55 & 97 & 95 & 0.98 & 0.37 & 0.78 \\
\hline 5095 & 14.992 & 0.939 & 2.07 & 98 & 98 & 1.12 & 0.67 & 0.80 \\
\hline 5242 & 14.880 & 0.697 & 9.36 & 98 & 98 & 1.09 & 0.28 & 0.77 \\
\hline 5268 & 15.317 & 0.663 & 6.32 & 98 & 91 & 1.05 & 0.06 & 0.58 \\
\hline 5309 & 14.965 & 0.706 & 4.39 & 98 & 98 & 1.10 & 0.24 & 0.72 \\
\hline 5332 & 15.047 & 0.672 & 2.74 & 98 & 98 & 1.08 & 0.13 & 0.46 \\
\hline
\end{tabular}


Table 3-Continued

\begin{tabular}{rcccccccr}
\hline \hline ID & $V$ & $(B-V)$ & $\begin{array}{c}\mathrm{R} \\
(\operatorname{arcmin})\end{array}$ & $\begin{array}{r}\mathrm{P}_{R V} \\
(\%)\end{array}$ & $\begin{array}{c}\mathrm{P}_{P M} \\
(\%)\end{array}$ & $\begin{array}{c}\mathrm{M}_{1} \\
\left(\mathrm{M}_{\odot}\right)\end{array}$ & $\begin{array}{c}\mathrm{M}_{2} \min \\
\left(\mathrm{M}_{\odot}\right)\end{array}$ & $\begin{array}{r}\mathrm{M}_{2} \\
\left(\mathrm{M}_{\odot}\right)\end{array}$ \\
\hline 5356 & 14.272 & 0.999 & 2.94 & 98 & 98 & 1.13 & 0.44 & 0.88 \\
5373 & 14.380 & 1.050 & 3.59 & 96 & 95 & $<1.14$ & 0.26 & $<0.87$ \\
5381 & 15.022 & 0.676 & 4.94 & 98 & 89 & 1.09 & 0.12 & 0.39 \\
5438 & 13.634 & 1.112 & 3.21 & 97 & 98 & $<1.14$ & 0.53 & $<0.96$ \\
5463 & 14.978 & 0.664 & 4.42 & 98 & 98 & 1.08 & 0.07 & 0.40 \\
5467 & 15.738 & 0.640 & 5.51 & 98 & 98 & $<1.02$ & 0.20 & $<0.74$ \\
5599 & 15.101 & 0.946 & 8.48 & 98 & 98 & $<1.13$ & 0.36 & $<0.80$ \\
5601 & 15.597 & 0.682 & 9.44 & 94 & 97 & 1.03 & 0.06 & 0.67 \\
5647 & 16.000 & 0.743 & 7.37 & 95 & 95 & 0.97 & 0.73 & 0.73 \\
5666 & 15.216 & 0.669 & 6.02 & 96 & 94 & 1.06 & 0.29 & 0.51 \\
5700 & 14.448 & 1.010 & 5.59 & 97 & 98 & 1.13 & 0.41 & 0.89 \\
5738 & 15.315 & 0.675 & 6.77 & 98 & 96 & 1.05 & 0.07 & 0.44 \\
5797 & 15.853 & 0.691 & 9.13 & 98 & 95 & 1.00 & 0.26 & 0.60 \\
5855 & 13.356 & 1.155 & 5.62 & 98 & 98 & $<1.14$ & 0.07 & $<0.99$ \\
5887 & 12.126 & 1.300 & 3.42 & 98 & 1 & $<1.14$ & 0.64 & $<1.13$ \\
6171 & 16.309 & 0.743 & 9.71 & 98 & 98 & $<0.94$ & 0.43 & $<0.68$ \\
6292 & 15.524 & 0.691 & 12.70 & 97 & 98 & 1.02 & 0.46 & 0.65 \\
6325 & 15.678 & 0.722 & 11.25 & 97 & 2 & 1.00 & 0.48 & 0.72 \\
6586 & 14.616 & 1.037 & 19.21 & 97 & 95 & $<1.13$ & 0.59 & $<0.85$ \\
8406 & 14.791 & 1.002 & 10.67 & 97 & 96 & 1.13 & 0.17 & 0.73 \\
\hline
\end{tabular}




\subsection{Double-Lined Orbital Solutions}

The RV measurements for the primary and secondary stars of a given SB2 binary are found using a TwO Dimensional CORelation (TODCOR) technique formulated by Zucker \& Mazeh (1994). TODCOR uses two template spectra to derive the two RVs of an SB2 binary simultaneously, greatly increasing our ability to recover reliable RVs even for those observations that appear highly blended in a one-dimensional cross-correlation function. As all of our detected SB2 binaries have mass ratios $\gtrsim 0.7$, we choose to use the same solar template that we use to derive RVs for all single stars and SB1 binaries as both template spectra in TODCOR. Our procedure in deriving the orbital solutions is to first solve for the orbit of the primary in the manner discussed in Section 3.1 and then use the derived orbital elements to solve for the full SB2 orbit (including the RVs of the secondary star). We provide the plotted orbital solutions in Figure 2; the plots are of the same format as for the SB1 binaries, except here, the primary RVs are plotted using filled circles while secondary RVs are plotted with open circles. Additionally, we present the tabulated orbital elements in Table 4 in similar format to Table 2, except here, in place of the mass function, we provide the quantity $m \sin ^{3} i$ and the mass ratio $(q)$.

We also include Table 5 that contains similar information on the SB2 binaries as we provide in Table 3 for the SB1 binaries. Here we do not quote a lower limit on the secondary mass as the mass ratio can be calculated directly from the orbital solution. We use the same photometric deconvolution procedure as for the SB1 binaries to derive the photometric mass estimates, except, here, we keep the mass ratio fixed. For the red-giant binary 3118, we cannot use this technique, as the system is observed to lie redward of the giant branch. Therefore, we use the Padova isochrone to formulate a mass-luminosity relation of $L \propto M^{11}$, valid for this region on the NGC 188 giant branch, to derive the appropriate correction to the observed $V$ magnitude, from which we can estimate the primary mass. (Specifically, we observe a mass ratio for 3118 of $q=0.795$, which implies a correction to the observed $V$ magnitude of $V_{1}=V+0.08$, and we use this $V_{1}$ to estimate the mass of the primary.) Given this primary mass estimate and the mass ratio, we can easily derive the secondary mass.

Again, we utilize a Monte Carlo technique to estimate the uncertainties on our mass estimates in a similar manner to Section 3.1. The mean uncertainty on the primary mass estimates is similar to that of the SB1 binaries. We can then use the mass ratio, primary mass and their respective uncertainties to derive a mean uncertainty on the secondary-mass estimates of $0.09 \mathrm{M}_{\odot}$, with a standard deviation about this mean of $0.02 \mathrm{M}_{\odot}$. Additionally, we utilize our SB2 binaries to check the accuracy of this photometric deconvolution technique by first estimating masses with the mass ratio fixed and then estimating masses for the same binaries without fixing the mass ratio (essentially, treating the systems as SB1 binaries and using the technique described in Section 3.1). For the primary mass, we find a mean difference between these two techniques of $0.01 \mathrm{M}_{\odot}$, and for the secondary mass estimates, we find a mean difference of $0.03 \mathrm{M}_{\odot}$. The standard deviations about these means are $0.02 \mathrm{M}_{\odot}$ and $0.06 \mathrm{M}_{\odot}$, respectively. These values lie within our estimated uncertainties, and demonstrate the robustness of the mass estimates for both SB1 and SB2 binaries derived using our photometric deconvolution technique. 

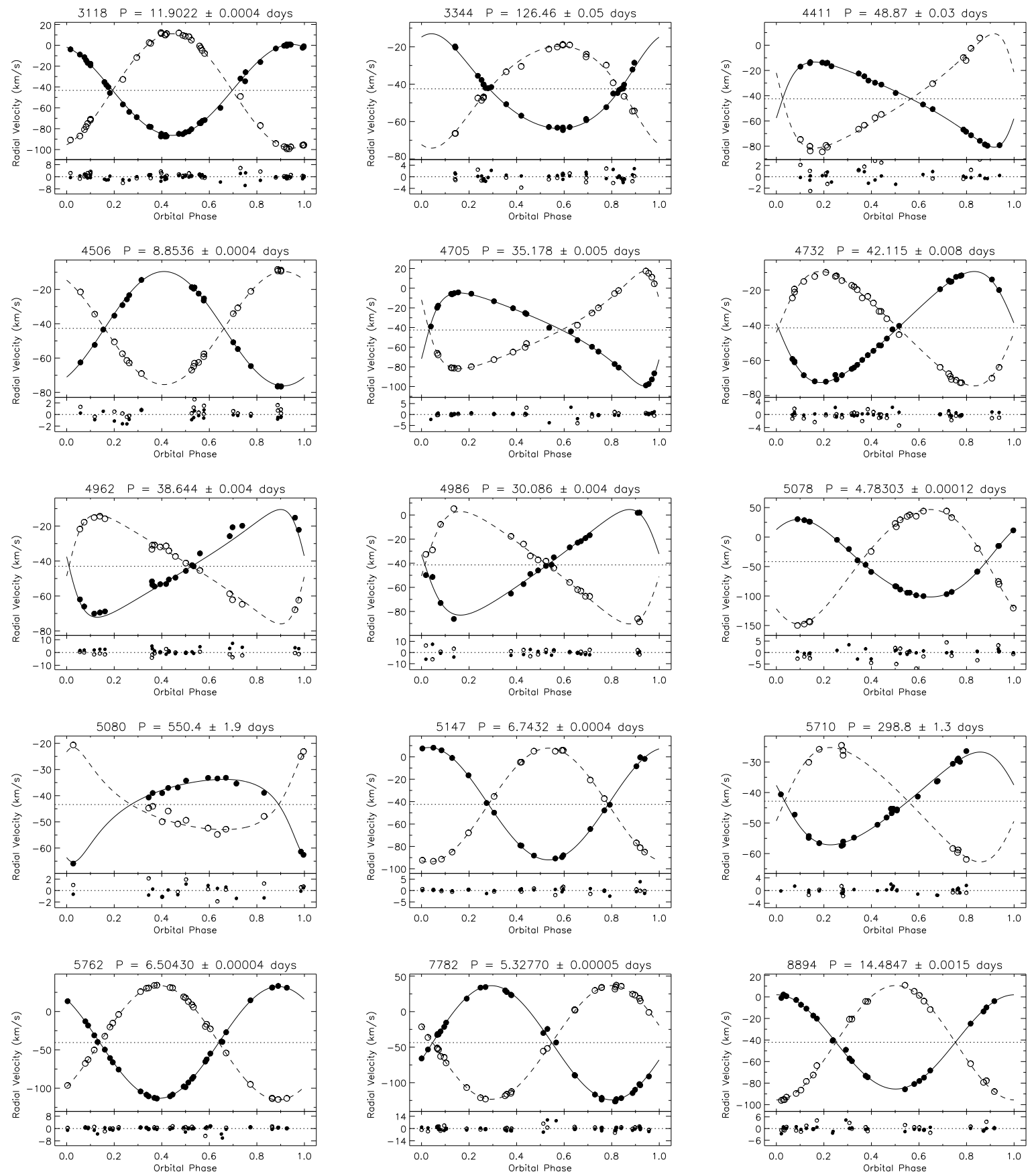

Fig. 2. - NGC 188 SB2 orbit plots. For each binary, we plot RV against orbital phase, showing the primary data points with filled circles and the secondary data points with open circles. The orbital fits to the data are plotted in the solid and dashed lines for the primary and secondary stars, respectively; the dotted line marks the $\gamma$-velocity. Beneath each orbit plot, we show the residuals from the fit. Above each plot, we give the binary ID and orbital period. 
Table 4. Orbital Parameters For NGC 188 Double-Lined Binaries

\begin{tabular}{|c|c|c|c|c|c|c|c|c|c|c|c|c|}
\hline ID & $\begin{array}{c}\mathrm{P} \\
\text { (days) }\end{array}$ & $\begin{array}{l}\text { Orbital } \\
\text { Cycles }\end{array}$ & $\begin{array}{c}\gamma \\
\left(\mathrm{km} \mathrm{s}^{-1}\right)\end{array}$ & $\begin{array}{c}\mathrm{K} \\
\left(\mathrm{km} \mathrm{s}^{-1}\right)\end{array}$ & $\mathrm{e}$ & $\begin{array}{c}\omega \\
(\operatorname{deg})\end{array}$ & $\begin{array}{c}\mathrm{T}_{\circ} \\
(\text { HJD-2400000 d) }\end{array}$ & $\begin{array}{c}\operatorname{asin} \mathrm{i} \\
\left(10^{6} \mathrm{~km}\right)\end{array}$ & $\begin{array}{l}\operatorname{msin}^{3} \mathrm{i} \\
\left(\mathrm{M}_{\odot}\right)\end{array}$ & $q$ & $\begin{array}{c}\sigma \\
\left(\mathrm{km} \mathrm{s}^{-1}\right)\end{array}$ & $\mathrm{N}$ \\
\hline 3118 & $\begin{array}{r}11.9022 \\
\pm 0.0004\end{array}$ & 133.8 & $\begin{array}{l}-43.23 \\
\pm 0.20\end{array}$ & $\begin{array}{r}43.6 \\
\pm 0.3 \\
54.9 \\
\pm 0.4\end{array}$ & $\begin{array}{r}0.011 \\
\pm 0.006\end{array}$ & $\begin{array}{r}20 \\
\pm 30\end{array}$ & $\begin{array}{r}50900.3 \\
\pm 1.0\end{array}$ & $\begin{array}{r}7.14 \\
\pm 0.06 \\
8.98 \\
\pm 0.07\end{array}$ & $\begin{array}{r}0.656 \\
\pm 0.012 \\
0.521 \\
\pm 0.009\end{array}$ & $\begin{array}{r}0.795 \\
\pm 0.009\end{array}$ & $\begin{array}{l}1.68 \\
1.98\end{array}$ & $\begin{array}{l}44 \\
36\end{array}$ \\
\hline 3344 & $\begin{array}{l}126.46 \\
\pm 0.05\end{array}$ & 24.2 & $\begin{array}{l}-42.51 \\
\pm 0.23\end{array}$ & $\begin{array}{r}25.4 \\
\pm 0.6 \\
27.3 \\
\pm 0.7\end{array}$ & $\begin{array}{r}0.175 \\
\pm 0.014\end{array}$ & $\begin{array}{l}338 \\
\pm 4\end{array}$ & $\begin{array}{r}50889.0 \\
\pm 1.6\end{array}$ & $\begin{array}{r}43.4 \\
\pm 1.1 \\
46.7 \\
\pm 1.3\end{array}$ & $\begin{array}{r}0.95 \\
\pm 0.06 \\
0.88 \\
\pm 0.05\end{array}$ & $\begin{array}{r}0.93 \\
\pm 0.03\end{array}$ & $\begin{array}{l}1.26 \\
1.70\end{array}$ & $\begin{array}{l}28 \\
20\end{array}$ \\
\hline 4411 & $\begin{array}{r}48.87 \\
\pm 0.03\end{array}$ & 11.8 & $\begin{array}{l}-42.4 \\
\pm 0.3\end{array}$ & $\begin{array}{r}33.7 \\
\pm 0.3 \\
45.3 \\
\pm 1.0\end{array}$ & $\begin{array}{r}0.433 \\
\pm 0.008\end{array}$ & $\begin{array}{r}251.6 \\
\pm 1.2\end{array}$ & $\begin{array}{r}50888.82 \\
\pm 0.21\end{array}$ & $\begin{array}{r}20.40 \\
\pm 0.16 \\
27.4 \\
\pm 0.6\end{array}$ & $\begin{array}{r}1.05 \\
\pm 0.05 \\
0.78 \\
\pm 0.03\end{array}$ & $\begin{array}{r}0.743 \\
\pm 0.018\end{array}$ & $\begin{array}{l}0.72 \\
2.55\end{array}$ & $\begin{array}{l}22 \\
15\end{array}$ \\
\hline 4506 & $\begin{array}{r}8.8536 \\
\pm 0.0004\end{array}$ & 115.7 & $\begin{array}{l}-42.69 \\
\pm 0.15\end{array}$ & $\begin{array}{r}33.3 \\
\pm 0.3 \\
32.9 \\
\pm 0.4\end{array}$ & $\begin{array}{r}0.007 \\
\pm 0.008\end{array}$ & $\begin{array}{r}210 \\
\pm 60\end{array}$ & $\begin{array}{r}50943.7 \\
\pm 1.4\end{array}$ & $\begin{array}{r}4.06 \\
\pm 0.04 \\
4.01 \\
\pm 0.05\end{array}$ & $\begin{array}{r}0.133 \\
\pm 0.004 \\
0.134 \\
\pm 0.003\end{array}$ & $\begin{array}{r}1.012 \\
\pm 0.015\end{array}$ & $\begin{array}{l}0.87 \\
1.04\end{array}$ & $\begin{array}{l}21 \\
20\end{array}$ \\
\hline 4705 & $\begin{array}{r}35.178 \\
\pm 0.005\end{array}$ & 25.7 & $\begin{array}{l}-42.53 \\
\pm 0.21\end{array}$ & $\begin{array}{r}47.3 \\
\pm 0.4 \\
49.5 \\
\pm 0.4\end{array}$ & $\begin{array}{r}0.487 \\
\pm 0.005\end{array}$ & $\begin{array}{l}245.4 \\
\pm 1.0\end{array}$ & $\begin{array}{r}50752.07 \\
\pm 0.07\end{array}$ & $\begin{array}{r}19.98 \\
\pm 0.22 \\
20.90 \\
\pm 0.21\end{array}$ & $\begin{array}{r}1.13 \\
\pm 0.03 \\
1.08 \\
\pm 0.03\end{array}$ & $\begin{array}{r}0.956 \\
\pm 0.013\end{array}$ & $\begin{array}{l}1.41 \\
1.33\end{array}$ & $\begin{array}{l}23 \\
20\end{array}$ \\
\hline 4732 & $\begin{array}{r}42.115 \\
\pm 0.008\end{array}$ & 17.6 & $\begin{array}{l}-41.46 \\
\pm 0.13\end{array}$ & $\begin{array}{r}31.56 \\
\pm 0.22 \\
32.5 \\
\pm 0.3\end{array}$ & $\begin{array}{r}0.232 \\
\pm 0.006\end{array}$ & $\begin{array}{r}86.3 \\
\pm 1.8\end{array}$ & $\begin{array}{r}50945.87 \\
\pm 0.20\end{array}$ & $\begin{array}{r}17.78 \\
\pm 0.13 \\
18.32 \\
\pm 0.20\end{array}$ & $\begin{array}{r}0.537 \\
\pm 0.013 \\
0.521 \\
\pm 0.009\end{array}$ & $\begin{array}{r}0.970 \\
\pm 0.013\end{array}$ & $\begin{array}{l}0.78 \\
1.29\end{array}$ & $\begin{array}{l}29 \\
28\end{array}$ \\
\hline 4962 & $\begin{array}{r}38.644 \\
\pm 0.004\end{array}$ & 80.4 & $\begin{array}{l}-43.0 \\
\pm 0.4\end{array}$ & $\begin{array}{r}30.8 \\
\pm 1.4 \\
31.3 \\
\pm 1.0\end{array}$ & $\begin{array}{r}0.445 \\
\pm 0.017\end{array}$ & $\begin{array}{r}83 \\
\pm 4\end{array}$ & $\begin{array}{r}51636.6 \\
\pm 0.3\end{array}$ & $\begin{array}{r}14.7 \\
\pm 0.7 \\
14.9 \\
\pm 0.5\end{array}$ & $\begin{array}{r}0.35 \\
\pm 0.03 \\
0.34 \\
\pm 0.04\end{array}$ & $\begin{array}{r}0.99 \\
\pm 0.06\end{array}$ & $\begin{array}{l}3.22 \\
1.91\end{array}$ & $\begin{array}{l}21 \\
19\end{array}$ \\
\hline 4986 & $\begin{array}{r}30.086 \\
\pm 0.004\end{array}$ & 103.3 & $\begin{array}{l}-41.4 \\
\pm 0.5\end{array}$ & $\begin{array}{r}43.8 \\
\pm 1.5 \\
46.6 \\
\pm 1.4\end{array}$ & $\begin{array}{r}0.342 \\
\pm 0.020\end{array}$ & $\begin{array}{r}82 \\
\pm 3\end{array}$ & $\begin{array}{r}53568.95 \\
\pm 0.25\end{array}$ & $\begin{array}{r}17.0 \\
\pm 0.7 \\
18.1 \\
\pm 0.7\end{array}$ & $\begin{array}{r}0.99 \\
+0.09 \\
0.93 \\
\pm 0.09\end{array}$ & $\begin{array}{r}0.94 \\
\pm 0.05\end{array}$ & $\begin{array}{l}3.22 \\
3.10\end{array}$ & $\begin{array}{l}18 \\
17\end{array}$ \\
\hline 5078 & $\begin{array}{r}4.78303 \\
\pm 0.00012\end{array}$ & 191.3 & $\begin{array}{l}-41.6 \\
\pm 0.3\end{array}$ & $\begin{array}{r}65.8 \\
\pm 0.4 \\
97.0 \\
\pm 1.0\end{array}$ & $\begin{array}{r}0.121 \\
\pm 0.006\end{array}$ & $\begin{array}{l}317 \\
\pm 3\end{array}$ & $\begin{array}{r}50704.13 \\
\pm 0.04\end{array}$ & $\begin{array}{r}4.30 \\
\pm 0.03 \\
6.34 \\
\pm 0.07\end{array}$ & $\begin{array}{r}1.25 \\
\pm 0.03 \\
0.846 \\
\pm 0.016\end{array}$ & $\begin{array}{r}0.678 \\
\pm 0.009\end{array}$ & $\begin{array}{l}1.41 \\
3.34\end{array}$ & $\begin{array}{l}23 \\
18\end{array}$ \\
\hline 5080 & $\begin{array}{l}550.4 \\
\pm 1.9\end{array}$ & 6.2 & $\begin{array}{l}-43.4 \\
\pm 0.3\end{array}$ & $\begin{array}{r}1.0 \\
15.8 \\
\pm 0.5 \\
15.7\end{array}$ & $\begin{array}{r}0.44 \\
\pm 0.03\end{array}$ & $\begin{array}{l}153 \\
\pm 4\end{array}$ & $\begin{array}{r}52273 \\
\pm 7\end{array}$ & $\begin{array}{r}107 \\
\pm 4 \\
107\end{array}$ & $\begin{array}{r}0.65 \\
\pm 0.09 \\
0.65\end{array}$ & $\begin{array}{r}1.01 \\
\pm 0.07\end{array}$ & $\begin{array}{l}0.95 \\
2.08\end{array}$ & $\begin{array}{l}14 \\
13\end{array}$ \\
\hline 5147 & 6.7432 & 66.5 & -42.45 & $\begin{array}{r} \pm 0.9 \\
50.2\end{array}$ & 0.012 & 350 & 54356.3 & $\begin{array}{r} \pm 7 \\
4.65\end{array}$ & $\begin{array}{r} \pm 0.07 \\
0.362\end{array}$ & 0.988 & 1.33 & 19 \\
\hline
\end{tabular}


Table 4-Continued

\begin{tabular}{|c|c|c|c|c|c|c|c|c|c|c|c|c|}
\hline ID & $\begin{array}{c}\mathrm{P} \\
\text { (days) } \\
\end{array}$ & $\begin{array}{l}\text { Orbital } \\
\text { Cycles }\end{array}$ & $\begin{array}{c}\gamma \\
\left(\mathrm{km} \mathrm{s}^{-1}\right) \\
\end{array}$ & $\begin{array}{c}\mathrm{K} \\
\left(\mathrm{km} \mathrm{s}^{-1}\right) \\
\end{array}$ & e & $\begin{array}{c}\omega \\
(\mathrm{deg})\end{array}$ & $\begin{array}{c}\mathrm{T}_{\circ} \\
(\text { HJD-2400000 d) } \\
\end{array}$ & $\begin{array}{c}\operatorname{asin} \mathrm{i} \\
\left(10^{6} \mathrm{~km}\right) \\
\end{array}$ & $\begin{array}{c}\operatorname{msin}^{3} \mathrm{i} \\
\left(\mathrm{M}_{\odot}\right) \\
\end{array}$ & $q$ & $\begin{array}{c}\sigma \\
\left(\mathrm{km} \mathrm{s}^{-1}\right) \\
\end{array}$ & $\mathrm{N}$ \\
\hline & \pm 0.0004 & & \pm 0.19 & $\begin{array}{r} \pm 0.4 \\
50.8 \\
\pm 0.3\end{array}$ & \pm 0.006 & \pm 30 & \pm 0.5 & $\begin{array}{r} \pm 0.04 \\
4.71 \\
\pm 0.03\end{array}$ & $\begin{array}{r} \pm 0.006 \\
0.357 \\
\pm 0.007\end{array}$ & \pm 0.011 & 0.96 & 17 \\
\hline 5710 & $\begin{array}{l}298.8 \\
\pm 1.3\end{array}$ & 4.1 & $\begin{array}{l}-42.86 \\
\pm 0.20\end{array}$ & $\begin{array}{r}15.2 \\
\pm 0.4 \\
18.7 \\
\pm 0.5\end{array}$ & $\begin{array}{r}0.215 \\
\pm 0.023\end{array}$ & $\begin{array}{r}74 \\
\pm 5\end{array}$ & $\begin{array}{r}50775 \\
\pm 4\end{array}$ & $\begin{array}{r}61.0 \\
\pm 1.7 \\
75.2 \\
\pm 2.2\end{array}$ & $\begin{array}{r}0.62 \\
\pm 0.04 \\
0.51 \\
\pm 0.03\end{array}$ & $\begin{array}{r}0.81 \\
\pm 0.03\end{array}$ & $\begin{array}{l}0.98 \\
1.08\end{array}$ & 26 \\
\hline 5762 & $\begin{array}{r}6.50430 \\
\pm 0.00004\end{array}$ & 421.7 & $\begin{array}{l}-40.48 \\
\pm 0.19\end{array}$ & $\begin{array}{r}72.9 \\
\pm 0.4 \\
74.6 \\
\pm 0.3\end{array}$ & $\begin{array}{r}0.004 \\
\pm 0.004\end{array}$ & $\begin{array}{r}40 \\
\pm 50\end{array}$ & $\begin{array}{r}51061.2 \\
\pm 1.0\end{array}$ & $\begin{array}{r}6.52 \\
\pm 0.04 \\
6.67 \\
\pm 0.03\end{array}$ & $\begin{array}{r}1.093 \\
\pm 0.013 \\
1.067 \\
\pm 0.015\end{array}$ & $\begin{array}{r}0.977 \\
\pm 0.008\end{array}$ & $\begin{array}{l}1.69 \\
1.26\end{array}$ & $\begin{array}{l}32 \\
29\end{array}$ \\
\hline 7782 & $\begin{array}{r}5.32770 \\
\pm 0.00005\end{array}$ & 426.5 & $\begin{array}{l}-43.6 \\
\pm 0.3\end{array}$ & $\begin{array}{r}80.4 \\
\pm 0.8 \\
80.0 \\
\pm 0.5\end{array}$ & $\begin{array}{r}0.013 \\
\pm 0.006\end{array}$ & $\begin{array}{r}250 \\
\pm 30\end{array}$ & $\begin{array}{r}52206.8 \\
\pm 0.4\end{array}$ & $\begin{array}{r}5.89 \\
\pm 0.06 \\
5.86 \\
\pm 0.04\end{array}$ & $\begin{array}{r}1.136 \\
\pm 0.020 \\
1.14 \\
\pm 0.03\end{array}$ & $\begin{array}{r}1.005 \\
\pm 0.013\end{array}$ & $\begin{array}{l}3.12 \\
2.12\end{array}$ & 32 \\
\hline 8894 & $\begin{array}{r}14.4847 \\
\pm 0.0015\end{array}$ & 43.5 & $\begin{array}{l}-42.11 \\
\pm 0.24\end{array}$ & $\begin{array}{r}43.7 \\
\pm 0.4 \\
53.1 \\
\pm 0.5\end{array}$ & $\begin{array}{r}0.008 \\
\pm 0.010\end{array}$ & $\begin{array}{r}360 \\
\pm 40\end{array}$ & $\begin{array}{r}51017.9 \\
\pm 1.8\end{array}$ & $\begin{array}{r}8.71 \\
\pm 0.09 \\
10.57 \\
\pm 0.12\end{array}$ & $\begin{array}{r}0.746 \\
\pm 0.019 \\
0.615 \\
\pm 0.015\end{array}$ & $\begin{array}{r}0.824 \\
\pm 0.012\end{array}$ & $\begin{array}{l}1.41 \\
1.78\end{array}$ & $\begin{array}{l}25 \\
22\end{array}$ \\
\hline
\end{tabular}


Table 5. Physical Properties of NGC 188 Double-Lined Binaries

\begin{tabular}{cccccccc}
\hline \hline ID & $V$ & $(B-V)$ & $\begin{array}{c}\mathrm{R} \\
(\operatorname{arcmin})\end{array}$ & $\begin{array}{c}\mathrm{P}_{R V} \\
(\%)\end{array}$ & $\begin{array}{c}\mathrm{P}_{P M} \\
(\%)\end{array}$ & $\begin{array}{c}\mathrm{M}_{1} \\
\left(\mathrm{M}_{\odot}\right)\end{array}$ & $\begin{array}{c}\mathrm{M}_{2} \\
\left(\mathrm{M}_{\odot}\right)\end{array}$ \\
\hline 3118 & 14.652 & 1.123 & 18.73 & 95 & 34 & 1.14 & 0.90 \\
3344 & 15.290 & 0.704 & 14.58 & 98 & 98 & 1.01 & 0.94 \\
4411 & 15.693 & 0.734 & 6.97 & 98 & 98 & 0.99 & 0.73 \\
4506 & 14.865 & 0.650 & 7.11 & 98 & 97 & 1.04 & 1.05 \\
4705 & 13.933 & 0.938 & 3.16 & 98 & 98 & 1.14 & 1.09 \\
4732 & 15.467 & 0.720 & 4.07 & 96 & 98 & 0.97 & 0.94 \\
4962 & 15.286 & 0.692 & 2.21 & 98 & 98 & 0.99 & 0.98 \\
4986 & 15.274 & 0.671 & 1.42 & 96 & 97 & 1.03 & 0.97 \\
5080 & 14.624 & 0.668 & 1.55 & 96 & 98 & 1.02 & 1.02 \\
5147 & 15.343 & 0.701 & 4.58 & 98 & 98 & 0.98 & 0.96 \\
5710 & 14.823 & 0.726 & 7.50 & 98 & 98 & 1.11 & 0.90 \\
5762 & 14.759 & 0.673 & 8.00 & 66 & 97 & 1.03 & 1.00 \\
8894 & 15.290 & 0.684 & 15.95 & 98 & 91 & 1.05 & 0.87 \\
\hline
\end{tabular}




\section{Binaries of Note}

In the following section, we discuss the properties of various intriguing binaries that we have discovered in NGC 188. We first discuss three binaries that contain potential encounter products. We then include our photometric variables and X-ray sources, and present evidence that 5015 is in fact a quadruple system composed of two SB1 binary cluster members.

\subsection{Binaries Containing Potential Encounter Products}

5078: 5078 has a period of $4.78303 \pm 0.00012$ days, well below the circularization period of 14.5 days in NGC 188 (Meibom \& Mathieu 2005). However this binary has a significantly higher than circular eccentricity, at $0.121 \pm 0.006$. 5078 is a particularly intriguing binary as it is a BS with an SB2 orbital solution. This relatively high eccentricity may be a sign of a recent dynamical interaction or an additional companion (Mazeh 1990). Triple systems are not uncommon within binary populations, with observational evidence ranging from 5-50\% (Mavor \& Mazeh 1987; Duquennoy \& Mavor 1991; Pourbaix et al. 2004; Tokovinin et al. 2006). Furthermore, Tokovinin et al. (2006) showed that for solar-type binaries, the frequency of additional companions increases towards shorter inner-binary periods, finding a frequency of tertiary companions for binaries with periods $\sim 5$ days of $\sim 65 \%$.

5080 : 5080 is a SB2 binary found right above the main-sequence turnoff at $V=14.624$ and $(B-V)=$ 0.668 . The system is located at 0.7 core radii from the cluster center, and has a $\mathrm{P}_{R V}=96 \%$ and a $\mathrm{P}_{P M}=$ $98 \%$. From our orbital solution, we find a mass ratio of $1.01 \pm 0.07$, and we estimate that both stars have masses of $\sim 1.02 \mathrm{M}_{\odot}$. However, from inspection of the cross-correlation functions, it is clear that the two stars have different luminosities. We checked for a potential template mismatch using a set of solar-metallicity synthetic spectral templates ranging from a $0.5 \mathrm{M}_{\odot}$ main-sequence star to a $1.14 \mathrm{M}_{\odot}$ star at the tip of the giant branch. For all spectra of 5080 in which we detect the secondary, a combination of two solar templates returns the highest two-dimensional correlation peak height and therefore the best fit to the data. Hence we proceed to use our standard solar spectrum as the template for both the primary and secondary stars in order to derive the luminosity ratio. The majority of the correlation functions are highly blended. Consequently we ran TODCOR on the four observations that show the largest RV separations and derive a luminosity ratio $\left(L_{2} / L_{1}\right)$ of 0.32 , with a standard deviation of 0.04 . Thus the secondary star appears to be under-luminous for its mass. We note that, if we take the lowest value for the mass ratio allowed by the error, of 0.94 , then we could be observing a binary containing a primary star that has evolved just past the turnoff with a main-sequence secondary star. If we take the mass of the primary star to be $1.02 \mathrm{M}_{\odot}$, as derived in Section 3.2 then the secondary star could have a mass as low as $0.96 \mathrm{M}_{\odot}$. Using these values with the Padova isochrone, we derive a luminosity ratio of 0.65 , which is certainly much larger than what we observe.

7782 : 7782 is a BS SB2 binary located at 9.7 core radii with a $\mathrm{P}_{R V}=95 \%$ and a $\mathrm{P}_{P M}=11 \% .7782$ is the second bluest of our detected BSs in NGC 188 with a $(B-V)=0.494$. Interestingly, we find the system to have a mass ratio of $1.005 \pm 0.013$, meaning that both stars in the system are likely more massive than the main-sequence turnoff mass. Utilizing TODCOR, we select the 11 observations with well separated peaks to

find a luminosity ratio of 0.739 with a standard deviation of 0.026 . We suggest that 7782 may be a BS - BS binary system. 


\subsection{Photometric Variables and X-ray Sources}

4289 : 4289 is a SB1 binary found at the base of the giant branch at a radius of 2.5 core radii. The binary is a secure cluster member with both $\mathrm{P}_{R V}$ and $\mathrm{P}_{P M}=98 \%$. We derive an orbital solution with a period of $11.4877 \pm 0.0009$ days and an eccentricity consistent with circular of $0.012 \pm 0.010$. We estimate that the primary star is likely a red giant with a mass of $<1.12 \mathrm{M}_{\odot}$, and the secondary star is on the main sequence with a mass of $<0.78 \mathrm{M}_{\odot}$. This binary was observed to be one of the brightest X-ray sources, GX28, in the Gondoin (2005b) survey. They point out that one would not expect a giant star in NGC 188 to show rapid rotation or surface activity unless the star is a member of a tight binary system in which rapid rotation has been maintained by synchronization. We do not see any evidence for line broadening due to rotation in our spectra, which corresponds to an upper limit of $\sim 10 \mathrm{~km} \mathrm{~s}^{-1}$ (derived from similar analysis to that of Rhode. Herbst \& Mathieu (2001)). With a period of $\sim 11.5$ days and assuming an appropriate radius for the primary star of $\sim 2.3 \mathrm{R}_{\odot}$, we would expect a maximum rotational velocity of $\sim 10 \mathrm{~km} \mathrm{~s}^{-1}$ resulting from tidal synchronization. According to Gondoin (2005a), even this relatively slow rotation may be sufficient to increase the surface coverage of magnetic-loop structures in giants like 4289 enough to produce the observed X-ray emission.

4705 : This SB2 binary is found at 1.3 core radii, and lies near the giant branch with $V=13.933$ and $(B-V)=0.938$. The binary is a high-probability cluster member with both $\mathrm{P}_{R V}$ and $\mathrm{P}_{P M}=98 \%$. We derive a kinematic orbital solution with a period of $35.178 \pm 0.005$ days and an eccentricity of $0.487 \pm$ 0.005. This star was observed as a photometric variable, V11, by Kaluzny (1990), who noted a dimming of almost 0.4 magnitudes over the course of the night of December 13, 1986. Kaluzny et al. conjecture that this variability and the location of 4705 on the CMD can be explained if 4705 is an eclipsing binary with a relatively unevolved red-giant primary and an upper-main-sequence secondary star. The observed photometric dimming occurred at a phase of $\sim 0.02$ in our derived orbit (when the RVs of both the primary and secondary stars were very near the $\gamma$-velocity of the system). We used the program NIGHTFALI7 to determine the phase at which one would expect to observe an eclipse in this system, and find that we would indeed expect an eclipse to occur at a phase of $\sim 0.02$. Thus 4705 may be an eclipsing binary system in NGC 188. Furthermore, we estimate the primary mass to be $1.14 \mathrm{M}_{\odot}$ and find a mass ratio of $0.956 \pm 0.013$. This would allow for an upper main-sequence secondary star as predicted. Additionally, 4705 was found to be an X-ray variable, GX18, by Gondoin (2005b), who observed low-amplitude brightness variations on the time scale of weeks. They suggest that these variations are due to slow rotation, as rotating giants can produce high X-ray luminosities, possibly related to the existence of magnetic fields induced by turbulent motion in their deepening convective zones. It has also been suggested by Zhang et al. (2002) and Gondoin (2005b) that 4705 may be an RS CVn system.

5379 : This SB1 BS binary lies at 1.6 core radii from the cluster center and is a secure cluster member with both $\mathrm{P}_{P M}$ and $\mathrm{P}_{R V}=98 \%$. This binary is a BS, with a $V$ magnitude of 15.373 and a $(B-V)$ color of 0.542 . We derive a period of $120.21 \pm 0.04$ days with an eccentricity of $0.24 \pm 0.03$. Additionally, Kafka \& Honeycutt (2003) found this binary to be a photometric variable (WV3) with a period of 0.18148 days. We cannot derive a kinematic orbital solution with this short period. We do observe signs of above average rotation in the 5379 spectra, and we have used the procedure of Rhode. Herbst \& Mathieu (2001) to

\footnotetext{
${ }^{7}$ NIGHTFALL is copyright (c) 1998-2002 Rainer Wichmann, (c) 2001-2002 Markus Kuster, (c) 2001- 2002 Patrick Risse and can be downloaded from http://www.hs.uni-hamburg.de/DE/Ins/Per/Wichmann/Nightfall.html
} 
derive a $v \sin i$ of $15.4 \pm 0.5 \mathrm{~km} \mathrm{~s}^{-1}$. If this photometric variability is due to chromospheric activity or star spots at this short period, we would expect a rotational velocity for the star of $>250 \mathrm{~km} \mathrm{~s}^{-1}$, which can be ruled out for all inclination angles greater than $\sim 3.5^{\circ}$. Kafka \& Honeycutt (2003) suggested that 5379 may be a member of the short-period end of the NGC $188 \mathrm{~W}$ UMa population. This now seems less likely given our lack of observed rapid rotation. We note that the photometric period, amplitude of the oscillations, and the observed $v \sin i$ lie within the observed range of $\delta$ Sct variable stars (Rodríguez et al. 2000). However 5379 does not lie near the instability strip.

5762 : 5762 is a SB2 binary found at the main-sequence turnoff at 3.4 core radii from the cluster center. The binary has a $\mathrm{P}_{P M}=97 \%$ and $\mathrm{P}_{R V}=66 \%$. We derive a circular orbit with a period of $6.50430 \pm$ 0.00004 days, a mass ratio near unity of $0.977 \pm 0.008$, and a minimum separation between the primary and secondary of $18.95 \pm 0.08 \mathrm{R}_{\odot}$. Zhang et al. $(2002,2004)$ identified this system as an eclipsing binary (V12). The observed photometric eclipse in Zhang et al. (2002) occurred at a phase of 0.88 in our orbital solution, when both stars in the system were moving near the $\gamma$-velocity. This provides further evidence for the eclipsing nature of the system. Meibom et al. (2009) discuss this eclipsing binary in detail. We simply point out that even if we are viewing this system at a low inclination angle, the true separation between the two stars will likely be very favorable to mass transfer as both stars evolve up the giant branch. As such, 5762 may be a pre-mass-transfer system which could represent a BS precursor.

\subsection{A Possible Quadruple System : 5015}

5015 is a $90 \%$ PM member, and upon preliminary inspection of the observed spectra and the resulting cross-correlation functions, we presumed that 5015 was a typical SB2 binary. There are two clear peaks in most of the 1D correlation functions, and both RVs are easily recovered using TODCOR for all but one observation. We followed the usual procedure of fitting an orbital solution to the primary, then using the derived orbital parameters to fit the full orbital solution, including the secondary velocities. However, we were unable to derive an SB2 orbit using the parameters from the fit to the primary. We then proceeded to fit a separate orbital solution to the secondary RVs, and found that the two solutions had entirely different parameters. We show the individual orbits in Figure 4.3 and give the respective orbital parameters in Table 6 . Individually, each of the derived $\gamma$-velocities results in a $\mathrm{P}_{R V}=0 \%$. Interestingly, though, if we take the average of the two $\gamma$-velocities, we get $-41.9 \pm 0.3 \mathrm{~km} \mathrm{~s}^{-1}$, which is very close to the cluster mean RV of $-42.36 \pm 0.04 \mathrm{~km} \mathrm{~s}^{-1}$ (Paper 1). Thus we have two options: either the two observed binaries are a chance superposition of two field binaries, or we are observing a quadruple system that is a likely member of NGC 188.

If we assume that the two binaries are not cluster members, then we can ask what is the likelihood that we are observing a superposition of two binaries in the field. To answer this question, we utilized the theoretical Besançon model of the Milky Way (Robin et al. 2003) to derive the expected number of field stars within one square degree, covering our observed magnitude range, towards the direction of NGC 188. We then assume that the locations of these field stars are described by a Poisson distribution and proceed to calculate the conditional probability that we would observe two field stars within a three arcsecond diameter fiber, given that we observe at least one, and find a $0.04 \%$ probability. Furthermore, since 5015 contains two binaries within a three arcsecond diameter region, we then multiply this value twice by the field binary fraction of 51\%, as observed by Duquennov \& Mavor (1991). Finally, we must account for the RVs of the two binaries. To do so, we again use the Besançon model to calculate the percentage of field stars with RVs 

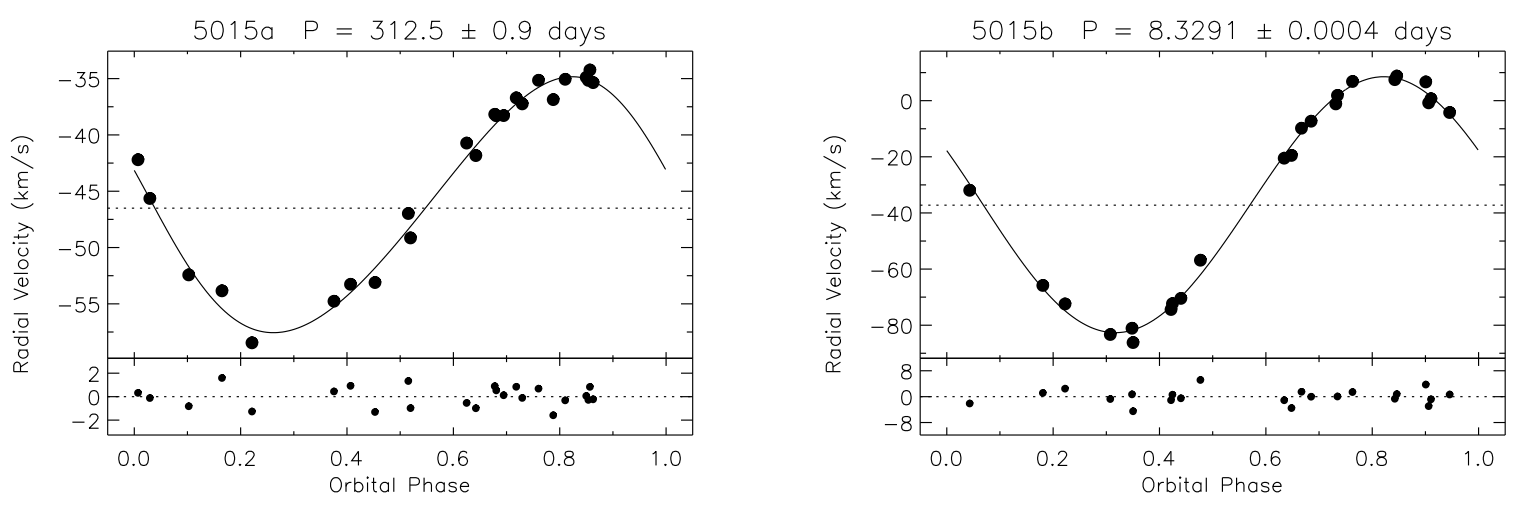

Fig. 3. - SB1 orbital solutions for the two binaries 5015a (left) and 5015b (right) that likely reside in a quadruple system. In the top panels we plot the observed data with dots and the orbital fits in the solid lines; the dotted lines mark the $\gamma$-velocities. Below the orbital plots, we show the RV residuals, and above the plots we provide the IDs and periods.

within five $\mathrm{km} \mathrm{s}^{-1}$ from the mean RV for NGC 188 (i.e., only including field stars with $-47 \mathrm{~km} \mathrm{~s}^{-1} \leq \mathrm{RV} \leq$ $37 \mathrm{~km} \mathrm{~s}^{-1}$ ), and find these stars to populate $20 \%$ of the field towards NGC 188. Including these constraints, the probability of observing two field binaries in the direction of NGC 188 within a three arcsecond diameter fiber that have RVs within five $\mathrm{km} \mathrm{s}^{-1}$ from the mean RV for NGC 188 is decidedly small, at $0.002 \%$. To date, we have observed a total of 1116 stars in the direction of NGC 188. Though this is a relatively large number of stars, it is certainly not enough for us to expect to observe such a chance superposition of two field binaries. Therefore, this scenario seems unlikely.

Conversely, we can assume that these two binaries are members of a quadruple system in which the two binaries orbit each other about the system's center of mass. Observations of field solar-type binary populations find the frequency of triples and higher-order systems to be 5-50\% (e.g. Mavor \& Mazeh 1987; Duquennoy \& Mavor 1991; Tokovinin 1997). Additionally, there is observational evidence for the presence of multiple-star systems in a few well studied open clusters (e.g., M67, Mathieu, Latham \& Griffin (1990); Praesepe, Mermilliod. Duquennov \& Mavor (1994); Pleiades, Bouvier. Rigaut \& Nadeau (1997); Hyades, Patience et al. (1998)). Recent $N$-body simulations by Hurley et al. (2005) suggest that in an old open

Table 6. Orbital Parameters for 5015a and 5015b

\begin{tabular}{lcc}
\hline \hline & $5015 \mathrm{a}$ & $5015 \mathrm{~b}$ \\
\hline $\mathrm{P}($ days $)$ & $312.5 \pm 0.9$ & $8.3291 \pm 0.0004$ \\
$\gamma\left(\mathrm{km} \mathrm{s}^{-1}\right)$ & $-46.50 \pm 0.24$ & $-37.2 \pm 0.6$ \\
$\mathrm{~K}\left(\mathrm{~km} \mathrm{~s}^{-1}\right)$ & $11.4 \pm 0.4$ & $45.6 \pm 0.7$ \\
$\mathrm{e}$ & $0.10 \pm 0.03$ & $0.008 \pm 0.016$ \\
$\omega(\mathrm{deg})$ & $74 \pm 21$ & $70 \pm 150$ \\
$\mathrm{~T}_{\circ}(\mathrm{HJD}-2400000 \mathrm{~d})$ & $51599 \pm 19$ & $51875 \pm 4$ \\
$\mathrm{asin} \mathrm{i}\left(10^{6} \mathrm{~km}\right)$ & $48.6 \pm 1.5$ & $5.22 \pm 0.08$ \\
$\mathrm{f}(\mathrm{m})\left(\mathrm{M}_{\odot}\right)$ & $4.7 \mathrm{e}-2 \pm 0.4 \mathrm{e}-2$ & $8.2 \mathrm{e}-2 \pm 0.4 \mathrm{e}-2$ \\
$\sigma\left(\mathrm{km} \mathrm{s}^{-1}\right)$ & 1.0 & 2.55 \\
$\mathrm{~N}$ & 23 & 22 \\
\hline
\end{tabular}


cluster, we might expect up to $\sim 7 \%$ of the sources to reside in dynamically-formed triple or higher-order systems. Thus we should not be surprised to find a few such star systems in NGC 188.

Using TODCOR, we derive a luminosity ratio of $0.36 \pm 0.02$. From the Padova isochrone, we find a luminosity ratio of $L \propto M^{4.5}$, valid for this region of the NGC 188 main-sequence, which results in a mass ratio of $0.80 \pm 0.04$. Therefore, the true center-of-mass RV of the quadruple system would be $-42.4 \pm 0.3$

$\mathrm{km} \mathrm{s}^{-1}$, which would result in a $\mathrm{P}_{R V}=98 \%$. This along with the Platais et al. (2003) $\mathrm{P}_{P M}=90 \%$ provides strong evidence for cluster membership.

\section{Summary}

In this paper, we present 98 binary orbits resulting from our ongoing RV survey of the old open cluster NGC 188. This is the second paper in a series aimed at characterizing the solar-type single- and binary-star populations within the cluster. These data will enable us to investigate the formation mechanisms and evolution of anomalous stars, like BSs, as they are influenced by the binary population, through comparison with detailed theoretical models of the cluster.

We provide our complete current RV database for NGC 188 in Table 1 including the measured RVs for all stars observed in the direction of NGC 188 over the course of our RV survey of the cluster. We use these data to derive the 70 SB1 (Section 3.1) and 15 SB2 (Section 3.2) orbital solutions for the NGC 188 cluster member binaries presented in this paper, and provide the results both graphically and as tabulated orbital elements. For the main-sequence, sub-giant and giant binaries we use a photometric deconvolution technique to estimate the masses of the primary and secondary stars relative to a 7 Gyr solar-metallicity isochrone, and we provide the SB1 results in Table 3 and the SB2 results in Table 5 For SB1 systems, we also provide a lower limit on the secondary mass, derived using the orbital mass function.

In Section 4 we identify a few binaries of note, including a likely quadruple system, 5015. Notably, 4705 and 5762 are both SB2 systems that may also be eclipsing binaries (5762 is studied in detail by Meibom et al. (2009)). We also observe the BS 7782 as an SB2 system with a mass-ratio near unity, which suggests that the system may contain two BS stars. We use TODCOR to investigate the luminosity ratio for the equal mass SB2 binary 5080 and find that the secondary star appears to be under-luminous for its mass. Finally we discuss the additional photometric variables and X-ray sources that are in binaries in NGC 188. The binaries of note discussed in Section 4 are ripe for further study.

The WIYN Open Cluster Study will continue its survey of NGC 188 in order to provide orbital solutions for all binaries in the cluster out to periods of 1000 days as well as a fraction of longer period binaries. In future papers, we will analyze the binary distribution in period, eccentricity and secondary mass, and constrain the cluster binary fraction. These data will form critical constraints on future detailed $N$-body models of NGC 188 as well as other open clusters, allowing us to study the complex interplay of stellar evolution and dynamics amongst the single- and binary-cluster members as they interact in the open cluster environment.

The authors would like to express their gratitude to the staff of the WIYN Observatory without whom we would not have been able to acquire these thousands of superb stellar spectra. We also thank the many undergraduate and graduate students who have helped to obtain these spectra over the years at WIYN for this project. We would like to acknowledge R. F. Griffin and J. E. Gunn for contributing their NGC 188 RVs to our project, who, in turn, wish to express their thanks to the Palomar Observatory for the use of the $5 \mathrm{~m}$ telescope. Thanks to Murray Fletcher for his expertise in developing the DAO RVS instrument, and 
to Jim Hesser who acquired a portion of the DAO NGC 188 data. Finally, we wish to thank to anonymous referee for the helpful suggestions in improving this paper. This work was funded by the National Science Foundation grant AST-0406615 and the Wisconsin Space Grant Consortium.

Facilities: WIYN 3.5m, DAO 1.2m, Palomar 5m

\section{A. APPENDIX}

\section{A.1. Field Binaries}

In our survey to find binary cluster members, we have serendipitously derived orbital solutions for 13 field binaries, all with either $\mathrm{P}_{R V}$ or $\mathrm{P}_{P M}=0 \%$. We note that some of these binaries appear to be kinematic members of NGC 188 from either PM or RV evidence, but none are cluster members in all three dimensions. In the interest of studies of the field binary population, we present these orbital plots (Figures 4 and 5) and parameters (Tables 7 and 8 ) here.
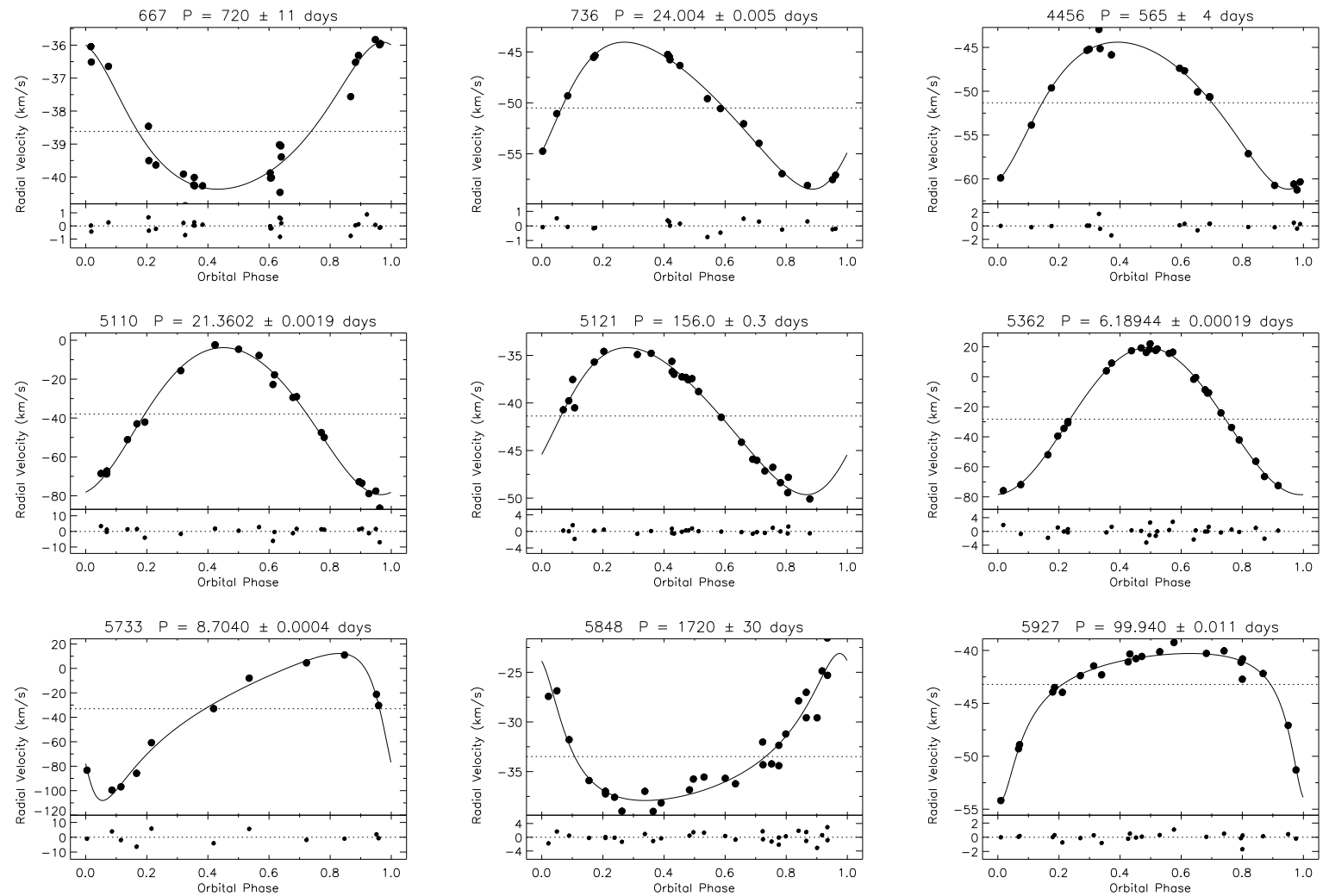

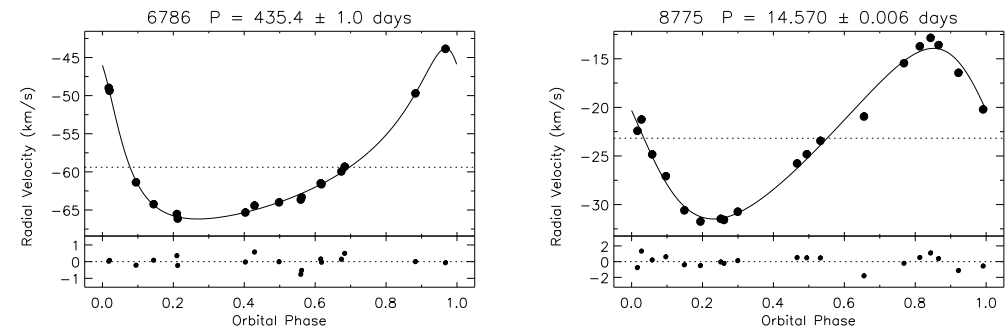

Fig. 4. - Field SB1 orbit plots. For each binary, we plot RV against orbital phase, showing the data points with black dots and the orbital fit to the data with the solid line; the dotted line marks the $\gamma$-velocity. Beneath each orbit plot, we show the residuals from the fit. Above each plot, we give the binary ID and orbital period.
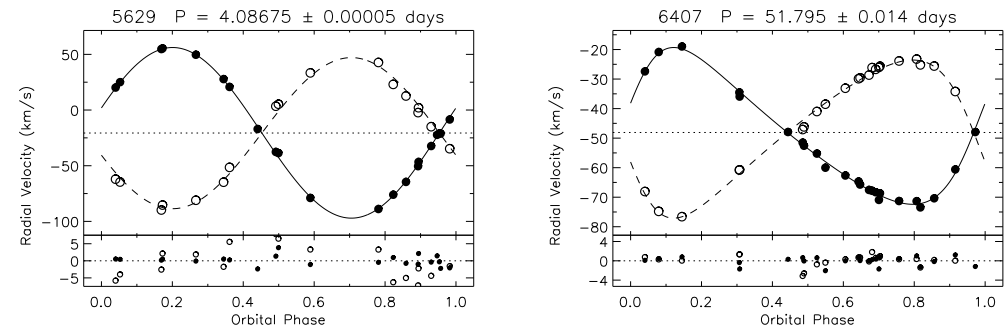

Fig. 5. - Field SB2 orbit plots. For each binary, we plot RV against orbital phase, showing the primary data points with filled circles and the secondary data points with open circles. The orbital fits to the data are plotted in the solid and dashed lines for the primary and secondary stars, respectively; the dotted line marks the $\gamma$-velocity. Beneath each orbit plot, we show the residuals from the fit. Above each plot, we give the binary ID and orbital period. 
Table 7. Orbital Parameters For Field Single-Lined Binaries

\begin{tabular}{|c|c|c|c|c|c|c|c|c|c|c|c|}
\hline ID & $\begin{array}{c}\mathrm{P} \\
\text { (days) }\end{array}$ & $\begin{array}{l}\text { Orbital } \\
\text { Cycles }\end{array}$ & $\left(\mathrm{km} \mathrm{s}^{-1}\right)$ & $\begin{array}{c}\mathrm{K} \\
\left(\mathrm{km} \mathrm{s}^{-1}\right)\end{array}$ & e & $\begin{array}{c}\omega \\
(\mathrm{deg})\end{array}$ & $\begin{array}{c}\mathrm{T}_{\circ} \\
(\mathrm{HJD}-2400000 \mathrm{~d})\end{array}$ & $\begin{array}{c}\operatorname{asin} \mathrm{i} \\
\left(10^{6} \mathrm{~km}\right)\end{array}$ & $\begin{array}{l}\mathrm{f}(\mathrm{m}) \\
\left(\mathrm{M}_{\odot}\right)\end{array}$ & $\left(\begin{array}{c}\sigma \\
\left(\mathrm{km} \mathrm{s}^{-1}\right)\end{array}\right.$ & $\mathrm{N}$ \\
\hline 667 & $\begin{array}{r}720 \\
\pm 11\end{array}$ & 2.3 & $\begin{array}{l}-38.61 \\
\pm 0.10\end{array}$ & $\begin{array}{r}2.29 \\
\pm 0.13\end{array}$ & $\begin{array}{r}0.22 \\
\pm 0.08\end{array}$ & $\begin{array}{r}16 \\
\pm 14\end{array}$ & $\begin{array}{r}52210 \\
\pm 30\end{array}$ & $\begin{array}{r}22.1 \\
\pm 1.3\end{array}$ & $\begin{array}{r}8.3 \mathrm{e}-4 \\
\pm 1.4 \mathrm{e}-4\end{array}$ & 0.47 & 26 \\
\hline 736 & $\begin{array}{r}24.004 \\
\pm 0.005\end{array}$ & 50.8 & $\begin{array}{l}-50.50 \\
\pm 0.11\end{array}$ & $\begin{array}{r}7.21 \\
\pm 0.16\end{array}$ & $\begin{array}{r}0.210 \\
\pm 0.019\end{array}$ & $\begin{array}{l}240 \\
\pm 6\end{array}$ & $\begin{array}{r}52039.8 \\
\pm 0.4\end{array}$ & $\begin{array}{r}2.33 \\
\pm 0.05\end{array}$ & $\begin{array}{r}8.7 \mathrm{e}-4 \\
\pm \quad 0.6 \mathrm{e}-4\end{array}$ & 0.42 & 17 \\
\hline 4456 & $\begin{array}{l}565 \\
\pm 4\end{array}$ & 2.9 & $\begin{array}{l}-51.32 \\
\pm 0.18\end{array}$ & $\begin{array}{r}8.4 \\
\pm 0.3\end{array}$ & $\begin{array}{r}0.20 \\
\pm 0.03\end{array}$ & $\begin{array}{r}207 \\
\pm 10\end{array}$ & $\begin{array}{r}52117 \\
\pm 15\end{array}$ & $\begin{array}{r}63.8 \\
\pm 2.1\end{array}$ & $\begin{array}{r}3.2 \mathrm{e}-2 \\
\pm 0.3 \mathrm{e}-2\end{array}$ & 0.75 & 18 \\
\hline 5110 & $\begin{array}{r}21.3602 \\
\pm 0.0019\end{array}$ & 163.2 & $\begin{array}{l}-38.0 \\
\pm 0.7\end{array}$ & $\begin{array}{r}37.8 \\
\pm 0.9\end{array}$ & $\begin{array}{r}0.10 \\
\pm 0.03\end{array}$ & $\begin{array}{r}195 \\
\pm 16\end{array}$ & $\begin{array}{r}51197.2 \\
\pm 0.9\end{array}$ & $\begin{array}{r}11.1 \\
\pm 0.3\end{array}$ & $\begin{array}{r}1.18 \mathrm{e}-1 \\
\pm 0.9 \mathrm{e}-2\end{array}$ & 3.07 & 22 \\
\hline 5121 & $\begin{array}{l}156.0 \\
\pm 0.3\end{array}$ & 7.3 & $\begin{array}{l}-41.37 \\
\pm 0.17\end{array}$ & $\begin{array}{r}7.7 \\
\pm 0.3\end{array}$ & $\begin{array}{r}0.16 \\
\pm 0.04\end{array}$ & $\begin{array}{r}243 \\
\pm 12\end{array}$ & $\begin{array}{r}51217 \\
\pm 5\end{array}$ & $\begin{array}{r}16.4 \\
\pm 0.7\end{array}$ & $\begin{array}{r}7.2 \mathrm{e}-3 \\
\pm \quad 0.9 \mathrm{e}-3\end{array}$ & 0.73 & 26 \\
\hline 5362 & $\begin{array}{r}6.18944 \\
\pm 0.00019\end{array}$ & 178.9 & $\begin{array}{l}-28.2 \\
\pm 0.3\end{array}$ & $\begin{array}{r}49.0 \\
\pm 0.5\end{array}$ & $\begin{array}{r}0.027 \\
\pm 0.009\end{array}$ & $\begin{array}{r}183 \\
\pm 20\end{array}$ & $\begin{array}{r}51035.3 \\
\pm 0.4\end{array}$ & $\begin{array}{r}4.17 \\
+0.04\end{array}$ & $\begin{array}{r}7.52 \mathrm{e}-2 \\
\pm 2.2 \mathrm{e}-3\end{array}$ & 1.51 & 29 \\
\hline 5733 & $\begin{array}{r}8.7040 \\
+0.0004\end{array}$ & 356.8 & $\begin{array}{l}-33.0 \\
\pm 2.2\end{array}$ & $\begin{array}{r}60 \\
\pm 5\end{array}$ & $\begin{array}{r}0.50 \\
\pm 0.05\end{array}$ & $\begin{array}{l}120 \\
\pm 7\end{array}$ & $\begin{array}{r}51606.69 \\
\pm 0.10\end{array}$ & $\begin{array}{r}6.2 \\
\pm 0.6\end{array}$ & $\begin{array}{r}1.3 \mathrm{e}-1 \\
\pm 0.3 \mathrm{e}-1\end{array}$ & 5.49 & 11 \\
\hline 5848 & $\begin{array}{l}1720 \\
\pm 30\end{array}$ & 2.0 & $\begin{array}{l}-33.5 \\
\pm 0.3\end{array}$ & $\begin{array}{r}7.4 \\
\pm 0.7\end{array}$ & $\begin{array}{r}0.45 \\
\pm 0.07\end{array}$ & $\begin{array}{r}26 \\
\pm 7\end{array}$ & $\begin{array}{r}51090 \\
\pm 24\end{array}$ & $\begin{array}{r}156 \\
\pm 15\end{array}$ & $\begin{array}{r}5.2 \mathrm{e}-2 \\
\pm 1.4 \mathrm{e}-2\end{array}$ & 1.48 & 29 \\
\hline 5927 & $\begin{array}{r}99.940 \\
\pm 0.011\end{array}$ & 113.5 & $\begin{array}{l}-43.21 \\
\pm 0.13\end{array}$ & $\begin{array}{r}6.9 \\
\pm 0.3\end{array}$ & $\begin{array}{r}0.600 \\
\pm 0.023\end{array}$ & $\begin{array}{l}165 \\
\pm 3\end{array}$ & $\begin{array}{r}48271.1 \\
\pm 0.6\end{array}$ & $\begin{array}{r}7.6 \\
\pm 0.4\end{array}$ & $\begin{array}{r}1.78 \mathrm{e}-3 \\
\pm 2.5 \mathrm{e}-4\end{array}$ & 0.62 & 23 \\
\hline 6786 & $\begin{array}{r}435.4 \\
\pm 1.0\end{array}$ & 3.7 & $\begin{array}{r}-59.39 \\
\pm 0.10\end{array}$ & $\begin{array}{r}11.19 \\
\pm 0.19\end{array}$ & $\begin{array}{r}0.491 \\
\pm 0.013\end{array}$ & $\begin{array}{r}36.7 \\
\pm 1.8\end{array}$ & $\begin{array}{r}51935.2 \\
\pm 1.4\end{array}$ & $\begin{array}{r}58.4 \\
\pm \quad 1.1\end{array}$ & $\begin{array}{r}4.18 \mathrm{e}-2 \\
\pm 2.2 \mathrm{e}-3\end{array}$ & 0.39 & 17 \\
\hline 8775 & $\begin{array}{r}14.570 \\
\pm 0.006\end{array}$ & 50.8 & $\begin{array}{r}-23.18 \\
\pm 0.22\end{array}$ & $\begin{array}{r}8.8 \\
\pm 0.3\end{array}$ & $\begin{array}{r}0.20 \\
+0.04\end{array}$ & $\begin{array}{r}74 \\
\pm 9\end{array}$ & $\begin{array}{r}50890.4 \\
\pm 0.3\end{array}$ & $\begin{array}{r}1.72 \\
\pm 0.06\end{array}$ & $\begin{array}{r}9.6 \mathrm{e}-4 \\
+1.0 \mathrm{e}-4\end{array}$ & 0.90 & 19 \\
\hline
\end{tabular}


Table 8. Orbital Parameters For Field Double-Lined Binaries

\begin{tabular}{|c|c|c|c|c|c|c|c|c|c|c|c|c|}
\hline ID & $\begin{array}{c}\mathrm{P} \\
\text { (days) }\end{array}$ & $\begin{array}{l}\text { Orbital } \\
\text { Cycles }\end{array}$ & $\begin{array}{c}\gamma \\
\left(\mathrm{km} \mathrm{s}^{-1}\right)\end{array}$ & $\begin{array}{c}\mathrm{K} \\
\left(\mathrm{km} \mathrm{s}^{-1}\right)\end{array}$ & $\mathrm{e}$ & $\begin{array}{c}\omega \\
(\mathrm{deg})\end{array}$ & $\begin{array}{c}\mathrm{T}_{\circ} \\
(\text { HJD-2400000 d) }\end{array}$ & $\begin{array}{c}\operatorname{asin} \mathrm{i} \\
\left(10^{6} \mathrm{~km}\right)\end{array}$ & $\begin{array}{c}\operatorname{msin}^{3} \mathrm{i} \\
\left(\mathrm{M}_{\odot}\right)\end{array}$ & $q$ & $\begin{array}{c}\sigma \\
\left(\mathrm{km} \mathrm{s}^{-1}\right)\end{array}$ & $\mathrm{N}$ \\
\hline 5629 & $\begin{array}{r}4.08675 \\
\pm 0.00005\end{array}$ & 237.0 & $\begin{array}{l}-20.6 \\
\pm 0.4\end{array}$ & $\begin{array}{r}76.6 \\
\pm 0.7 \\
67.8 \\
\pm 2.0\end{array}$ & $\begin{array}{r}0.007 \\
\pm 0.007\end{array}$ & $\begin{array}{r}290 \\
\pm 60\end{array}$ & $\begin{array}{r}51336.4 \\
\pm 0.7\end{array}$ & $\begin{array}{r}4.31 \\
\pm 0.04 \\
3.81 \\
\pm 0.12\end{array}$ & $\begin{array}{r}0.60 \\
\pm 0.04 \\
0.68 \\
\pm 0.03\end{array}$ & $\begin{array}{r}1.13 \\
\pm 0.04\end{array}$ & $\begin{array}{l}1.59 \\
5.14\end{array}$ & 21 \\
\hline 6407 & $\begin{array}{r}51.795 \\
\pm 0.014\end{array}$ & 19.8 & $\begin{array}{l}-48.08 \\
\pm 0.18\end{array}$ & $\begin{array}{r}26.5 \\
\pm 0.3 \\
26.8 \\
\pm 0.4\end{array}$ & $\begin{array}{r}0.289 \\
\pm 0.009\end{array}$ & $\begin{array}{l}286.8 \\
\pm 2.1\end{array}$ & $\begin{array}{r}50937.5 \\
\pm 0.3\end{array}$ & $\begin{array}{r}18.10 \\
\pm 0.24 \\
18.3 \\
\pm 0.3\end{array}$ & $\begin{array}{r}0.358 \\
\pm 0.013 \\
0.355 \\
\pm 0.011\end{array}$ & $\begin{array}{r}0.992 \\
\pm 0.021\end{array}$ & $\begin{array}{l}0.97 \\
1.27\end{array}$ & 24 \\
\hline
\end{tabular}




\section{REFERENCES}

Abt, H. A. \& Willmarth, D. W. 1999, ApJ, 521, 682

Belloni, T., Verbunt, F., \& Mathieu, R. D. 1998, A\&A, 339, 431

Bonatto, C., Bica, E. \& Santos Jr., J. F. C. 2005 A\&A, 433, 917

Bouvier, J., Rigaut, F. \& Nadeau, D. 1997, A\&A, 323, 139

Debernardi, Y., Mermilliod, J.-C., Carquillat, J.-M. \& Ginestet, N. 2000, A\&A, 354, 881

Duquennoy, A. \& Mayor, M. 1991, AA, 248, 485

Fornal, B., Tucker, D. L., Smith, J. A., Allam, S. S., Rider, C. J. \& Sung, H. 2007, AJ, 133, 1409

Geller, A. M., Mathieu, R. D., Harris, H. C. \& McClure, R. D. 2008, AJ, 135, 2264 (Paper 1)

Girardi, L., Bertelli, G., Bressan, A., Chiosi, C., Groenewegen, M. A. T., Marigo, P., Salasnich, B. \& Weiss, A. 2002, A\&A, 391, 195

Gondoin, P. 2005a, A\&A, 444, 531

Gondoin, P. 2005b, A\&A, 438, 291

Heggie, D. C. 1974, Proceedings of IAU Symposium 62, "The stability of the solar system and of small stellar systems", 225, ed. Kozai, Y.

Hurley, J. R., Pols, O. R., Aarseth, S. J. \& Tout, C. A. 2005, MNRAS, 363, 293

Hurley, J. R. \& Tout, C. A. 1998, MNRAS, 300, 977

Kafka, S. \& Honeycutt, R. K. 2003, AJ, 126276

Kaluźny, J. 1990, AcA, 40, 61

Mathieu, R. D. 2000, ASP, 198, 517

Mathieu, R. D., Latham, D. W. \& Griffin, R. F. 1990, AJ, 100, 1859

Mayor, M. \& Mazeh, T. 1987, A\&A, 171, 157

Mazeh, T. 1990, AJ, 99, 675

Meibom, S. Grundahl, F., Clausen, J.V., Mathieu, R.D., Nielson, T.B., Frandsen, S., Pigulski, A., Narwid, A., Steslicki, M. \& Lefever, K. 2009, AJ, 137, 5086

Meibom, S. \& Mathieu, R. D. 2005, ApJ, 620, 970

Mermilliod, J.-C., Duquennoy, A. \& Mayor, M. 1994, A\&A, 283, 515

Mermilliod, J.-C. \& Mayor, M. 1999, A\&A, 352, 479

Mermilliod, J.-C., Rosvick, J. M., Duquennoy, A. \& Mayor, M. 1992, 265, 513

Patience, J., Ghez, A. M., Reid, I. N., Weinberger, A. J. \& Matthews, K. 1998, AJ, 115, 1972 
Platais, I., Kozhurins-Platais, V., Mathieu, R. D., Girard, T. M. \& van Altena, W. F. 2003, AJ, 126, 2992

Pourbaix, D., Tokovinin, A. A., Batten, A. H., Fekel, F. C., Hartkopf, W. I., Levato, H., Morrell, N. I., Torres, G. \& Udry, S. 2004, A\&A, 424, 727

Rhode, K. L., Herbst, W. \& Mathieu, R. D. 2001, AJ, 122, 3258

Robin, A. C., Reylé, C., Derriére, S. \& Picaud, S. 2003, A\&A, 409,523

Rodríguez, E. López-González M. J. \& López de Copa, P. 2000, A\&AS, 144, 469

Stetson, P. B., McClure, R. D. \& VandenBerg, D. A. 2004, PASP, 116, 1012

Tokovinin, A. 1997, A\&AS, 124, 75

Tokovinin, A., Thomas, S., Sterzik, M. \& Udry, S. 2006, A\&A, 450, 681

Zhang, X.B., Deng, L., Tian, B. \& Zhou, X. 2002, AJ, 123, 1548

Zhang, X.B., Deng, L., Zhou, X. \& Xin, Y. 2004, MNRAS, 355, 1369

Zucker, S. \& Mazeh, T. 1994, ApJ, 420, 806

This preprint was prepared with the AAS LATEX macros v5.2. 